This item was submitted to Loughborough's Research Repository by the author.

Items in Figshare are protected by copyright, with all rights reserved, unless otherwise indicated.

\title{
Equations enforcing repetitions under permutations
}

PLEASE CITE THE PUBLISHED VERSION

https://doi.org/10.1016/j.dam.2020.05.026

PUBLISHER

ELSEVIER

VERSION

AM (Accepted Manuscript)

PUBLISHER STATEMENT

This paper was accepted for publication in the journal Discrete Applied Mathematics and the definitive published version is available at https://doi.org/10.1016/j.dam.2020.05.026

\section{LICENCE}

CC BY-NC-ND 4.0

\section{REPOSITORY RECORD}

Day, Joel, Pamela Fleischmann, Florin Manea, and Dirk Nowotka. 2020. "Equations Enforcing Repetitions Under Permutations”. Loughborough University. https://hdl.handle.net/2134/13090640.v1. 


\title{
Equations Enforcing Repetitions under Permutations
}

\author{
Joel D. Day \\ Loughborough University, Department of Computer Science, Loughborough, UK \\ Pamela Fleischmann \\ Kiel University, Department of Computer Science, Kiel, Germany \\ Florin Manea \\ University of Göttingen, Institute of Computer Science, Göttingen, Germany \\ Dirk Nowotka \\ Kiel University, Department of Computer Science, Kiel, Germany
}

\begin{abstract}
The notion of repetition of factors in words is central to combinatorics on words. A recent generalization of this concept considers repetitions under permutations: given an alphabet $\Sigma$ and a morphism or antimorphism $f$ on $\Sigma^{*}$, whose restriction to $\Sigma$ is a permutation, $w$ is an $[f]$-repetition if there exists $\gamma \in \Sigma^{*}$, an integer $k \geq 2$, and the positive integers $i_{1}, \ldots, i_{k}$ such that $w=f^{i_{1}}(\gamma) f^{i_{2}}(\gamma) \cdots f^{i_{k}}(\gamma)$. In this paper, we extend a series of classical repetition enforcing word equations to this general setting to obtain a series of word equations whose solutions are $[f]$-repetitions.
\end{abstract}

Keywords: Combinatorics on words, Word Equations, Lyndon-Schützenberger equation, Fine and Wilf's theorem, Pseudoperiodicity

2010 MSC: 68R15

\section{Introduction}

The study of repetitive sequences in words is one of the central topics of combinatorics on words, with applications in, e.g., pattern matching and stringology in general, or more specific in data compression and bioinformatics (see [1, 2]). Part of the inves5 tigations on this topic deals with repetition enforcing relations or equations. Intuitively, a word is a finite sequence of letters (symbols) from a finite set, called an alphabet. Basically, a repetition enforcing relation for words is a relation, or a statement, that is fulfilled only by words that can be expressed as repetitions (i.e., repeated concatenation)

Email addresses: J.Day@lboro.ac.uk (Joel D. Day), fpa@informatik.uni-kiel.de (Pamela Fleischmann), florin.manea@informatik.uni-goettingen.de (Florin Manea), dn@informatik.uni-kiel.de (Dirk Nowotka) 
of some (other) word. For instance, it is well known (see, e.g., [3]) that a word $w$ is a factor, other than a prefix (beginning of the word) or a suffix (end of the word), of the word $w w$ if and only if $w \in t\{t\}^{+}$for some shorter word $t$, i.e., $w$ is a repetition of at least two occurrences of the word $t$. Another prominent example of a repetition enforcing statement is the Theorem of Fine and Wilf [4]: considering two words $u$ and $v$ and the repetitions $\alpha=u^{\ell}$ and $\beta=v^{k}$ that share a common prefix of length at least

${ }_{15}|u|+|v|-\operatorname{gcd}(|u|,|v|)$, then both $u$ and $v$ are repetitions of some word $t$, i.e., $u, v \in\{t\}^{+}$. The equation of Lyndon and Schützenberger [5] is an example of a repetition enforcing equation: if $u^{\ell}=v^{m} w^{n}$, for some non-empty words $u, v, w$ and $\ell, m, n \geq 2$ then there exists a word $t$ (called the root) such that $u, v, w \in\{t\}^{+}$, so $u, v, w$ are all repetitions with the same root.

Pseudo-repetitions were introduced [6, 7], as a generalisation of classical repetitions, inspired by molecular biology. A word $w$ is a pseudo-repetition, or more precisely an $f$-repetition, if it equals a repeated concatenation of one of its prefixes $t$ and its image $f(t)$ under some morphism or antimorphism (for short "anti-/morphism") $f$, i.e. $w$ is in $t\{t, f(t)\}^{+}$. To fit the biological motivation, in [7] $f$ was defined as an antimorphic involution, i.e. $f^{2}(w)=w$ for all words $w$. More interesting to us, if $f$ is not restricted, pseudo-repetitions generalise not only repetitions (when $f$ is the identity morphism), but also palindromes (when $f$ is the mirror image); both these concepts are central in combinatorics on words, so their generalisations are of intrinsic theoretical interest. Initial results (see [7] and [8]) concerned generalisations of the Theorem of Fine and Wilf, of the Lyndon and Schützenberger equation, and of other repetition enforcing results to the setting of $f$-repetitions for antimorphic involutions $f$. For instance, Czeizler et al. [8] introduced a generalisation of Lyndon and Schützenberger's equations of a different kind. They considered equations of the form

$$
u_{1} u_{2} \cdots u_{\ell}=v_{1} v_{2} \cdots v_{m} w_{1} w_{2} \cdots w_{n}
$$

with $u_{i} \in\{u, f(u)\}$ for all $1 \leq i \leq \ell, v_{j} \in\{v, f(v)\}$ for all $1 \leq j \leq m$, and $w_{k} \in\{w, f(w)\}$ for all $1 \leq k \leq n$, and studied under which conditions the solutions $u, v, w$ of such an equation are all contained in $\{t, f(t)\}^{+}$, for some word $t$. That is, in other words, the conditions under which $u, v, w$ are necessarily generalised $f$ repetitions. A complete characterisation of the conditions under which this equation 25 has only $f$-repetitive solutions was obtained in [9].

Going a step further, the case of $f$-repetitions (over an alphabet $\Sigma$ ) for an anti/morphism $f$ that acts as a permutation on $\Sigma$ (anti-/morphic permutation) was considered in [10], where a series of results in the style of the Theorem of Fine and Wilf were given. Introduced in [10], but only briefly studied in that paper, was also a more general notion of repetition, that we will call here an $[f]$-repetition. If $f$ is an anti-/morphic permutation and $w=f^{i_{1}}(\gamma) f^{i_{2}}(\gamma) \cdots f^{i_{k}}(\gamma)$, for some $k \geq 2$, then $w$ is called [f]repetition of root $\gamma$, or for short $[f, \gamma]$-repetition. A variant of the Theorem of Fine and Wilf was shown for $[f]$-repetitions in the case when $f$ is a morphism. This notion also appears in a series of papers regarding avoidability of patterns under anti-/morphic 35 permutations: in [11] the avoidability of patterns of the form $\pi^{i}(x) \pi^{j}(x) \pi^{k}(x)$, i.e., $[\pi]$-cubes, for $\pi$ a variable that can be replaced by anti-/morphic permutations, was studied, while in [12] the avoidability of general $[\pi]$-repetitions was considered. Finally, algorithmic problems like deciding whether a word is an $f$-repetition [13, 14, 15] 
or whether a word contains $f$-repetitions [16, 17, 18] for different types of functions

(including anti-/morphic permutations) were investigated. However, we are not aware of any algorithmic results regarding $[f]$-repetitions.

In this paper we analyse a series of $[f]$-repetition enforcing word equations, for an anti-/morphic permutation $f$. That is, we analyse a series of word equations whose solutions are necessarily $[f]$-repetitions. We first analyse the case when $f$ is a morphic permutation, and we show that a series of classical repetition enforcing equations are extendible to this more general setting. For instance we show that for all integer numbers $a, b, c$, and $d$ both

$$
f^{a}(x) f^{b}(y)=f^{c}(y) f^{d}(x) \quad \text { and } \quad f^{a}(u) f^{b}(u)=x f^{c}(u) y
$$

with $x, y \neq \varepsilon$, enforce the words $x, y$, respectively $u$, that satisfy these equations, to be $[f]$-repetitions of a word $t$.

Our main result is an extension of the Lyndon-Schützenberger equation. More precisely, we consider the equation

$$
f^{a_{1}}(u) \ldots f^{a_{r}}(u) f^{b_{1}}(v) \ldots f^{b_{s}}(v)=f^{c_{1}}(w) \ldots f^{c_{t}}(w)
$$

parametrised by the numbers $r, s, t \geq 2$, and $a_{1}, \ldots, a_{r}, b_{1}, \ldots, b_{s}, c_{1} \ldots, c_{t}$. We show that if $u, v, w$ satisfy this equation then there exists a word $t$ such that each of $u, v$, and $w$ is in $\left\{t, f(t), \ldots, f^{\text {ord }(f)}(t)\right\}^{+}$. That is, each of these words is either an $[f]$-repetition of $t$, or it is the image of $t$ under some iteration $f^{i}$ of the morphic permutation $f$. This result is obtained as the conclusion of a thorough case analysis, structured as a series of technical lemmas, and presented in Section 3 These results complement the generalised

so Fine and Wilf Theorem obtained in this setting in [10].

In the case of $f$ antimorphic, we show, in Section 4, that the equation $f^{a}(u) f^{b}(u)=$ $x f^{c}(u) y$, parametrised by the non-empty words $x$ and $y$ and the integers $a, b, c$, may have as solutions words $u$ which are not $[f]$-repetitions. Thus, following the results of [8, 7], we characterise exactly the paramterized equations (or, in other words, find all the parameters/exponents $a_{1}, a_{2}, a_{3}, b_{1}$, and $b_{2}$ )

$$
f^{a_{1}}(u) f^{a_{2}}(u) f^{a_{3}}(u)=x f^{b_{1}}(u) f^{b_{2}}(u) y,
$$

with $x, y \neq \varepsilon$, whose solutions are [ $f$ ]-repetitions. We use this characterisation to show a result in the style of the Theorem of Fine and Wilf and to define a class of extensions of the Lyndon-Schützenberger equation that only have solutions which are $[f]$-repetitions.

The paper is organised as follows: we first give the basic definitions and recall some

55 preliminary results, then we present the results for the case when $f$ is a morphic permutation, and finally we present the results for when $f$ is an antimorphic permutation.

\section{Preliminaries}

Let $\mathbb{N}$ be the set of natural numbers and $\mathbb{Z}$ be the one of integer numbers. For convenience $\mathbb{N}_{0}$ is the set $\mathbb{N} \cup\{0\}$ and $\mathbb{N}_{\geq k}$ contains all natural numbers greater than or

6o equal to $k$. Moreover $[n]$ denotes the set $\{1, \ldots, n\}$ and $[n]_{0}$, analogously to $\mathbb{N}_{0}$, the set $[n] \cup\{0\}$ for an $n \in \mathbb{N}$. For $a, b \in \mathbb{Z}$ and $k \in \mathbb{N}$ define the congruence relation $\equiv_{k}$ such 
that $a \equiv_{k} b$ if and only if $k$ divides $a-b$. The quotient ring $\mathbb{Z} / \equiv_{k}=\mathbb{Z} / k \mathbb{Z}$ will be abbreviated by $\mathbb{Z}_{k}$ for $k \in \mathbb{Z}$. For $m, n \in \mathbb{N}$, let $\operatorname{gcd}(m, n)$ denote their greatest common divisor.

Regarding words, $\Sigma=\{\mathrm{a}, \mathrm{b}, \mathrm{c}, \ldots\}$ will be a finite alphabet and $\Sigma^{*}$ denotes the set of all words over $\Sigma$. The empty word is denoted by $\varepsilon$ and $\Sigma^{+}$is the free semi-subgroup $\Sigma^{*} \backslash\{\varepsilon\}$. Set additionally $\Sigma^{\leq k}:=\left\{w \in \Sigma^{*}|| w \mid \leq k\right\}$ where $|w|$ denotes the word's length. For two words $u$ and $v$ over $\Sigma$, set $d_{u, v}:=\operatorname{gcd}(|u|,|v|)$.

We say $u \in \Sigma^{*}$ is a factor of $w \in \Sigma^{*}$, if $w=x u y$ for some words $x, y \in \Sigma^{*}$. By this definition two special cases occur: $u$ is a prefix of $w$ if $x=\varepsilon$ and a suffix if $y=\varepsilon$. A word $u \in \Sigma^{*}$ is said to occur strictly inside (is a proper factor of) another word $w \in \Sigma^{*}$ if $u$ is a factor of $w$, other than a prefix or a suffix. Denote the set of a word's prefixes and suffixes by Pref and Suff respectively. For $w \in \Sigma^{*}$ every position $p \in \mathbb{N}_{\leq|w|}$ between two letters of $w$ is called a point of $w$.

$75 \quad$ The powers of a word $w \in \Sigma^{*}$ are defined recursively by $w^{0}=\varepsilon, w^{n}=w w^{n-1}$ for $n \geq 1$. If $w$ cannot be expressed as a power of another word, then $w$ is said to be primitive.

We say that $f: \Sigma^{*} \rightarrow \Sigma^{*}$ is a morphism (resp. antimorphism) if $f(x y)=f(x) f(y)$ (resp., $f(x y)=f(y) f(x)$ ), for any words $x, y \in \Sigma^{*}$. Note that, to define an antiso /morphism it is enough to give the definitions of $f(a)$ for all $a \in \Sigma$, and, in particular, for a bijective anti-/morphism we have that $|f(a)|=1$ for all $a \in \Sigma$. If $f$ is a bijective anti-/morphism then we call $f$ an anti-/morphic permutation. If $f$ is a permutation of $\Sigma$ then the order ord $(f)$ of $f$ is defined as the smallest positive number such that $f^{\operatorname{ord}(f)}(\mathrm{a})=\mathrm{a}$ for all $\mathrm{a} \in \Sigma$. In the morphic case, we have $f^{\operatorname{ord}(f)}(w)=w$ for all

${ }_{85} w \in \Sigma^{*}$, but considering the antimorphic permutation $f=(\mathrm{abc})$ on $\Sigma$, the identity is not reached after ord $(f)=3$ iterations because $f^{3}(\mathrm{ab})=$ ba. If $f$ is an antimorphic permutation then $f^{2 \operatorname{ord}(f)}(w)=w$ holds for all $w \in \Sigma^{*}$. This leads to the fact that the exponents of an anti-/morphic permutation $f$ can be considered to be elements of $\mathbb{Z}_{\operatorname{ord}(f)-1}$ resp. $\mathbb{Z}_{2 \operatorname{ord}(f)-1}$, i.e. $f^{a-b}$ is, for all $a, b \in \mathbb{Z}$, a well-defined iteration of $f$.

For an anti-/morphic permutation $f$, a word $w \in \Sigma^{*}$ is said to be an $[f]$-repetition if there exists $t \in \Sigma^{+}, k \geq 2$, and $a_{1}, \ldots, a_{k} \in \mathbb{Z}$ such that $w=f^{a_{1}}(t) f^{a_{2}}(t) \cdots f^{a_{k}}(t)$. In the case, that $t$ is the shortest such word, $t$ is called the root of the [f]-repetition $w$ and if it is important, $w$ will be called an $[f, t]$-repetition. If $w$ is not an $[f]$-repetition, then $w$ is $[f]$-primitive.

95 $\quad$ For instance, the word $w=$ abcaab is $\left[\mathrm{Id}_{\Sigma}\right]$-primitive, where $\mathrm{Id}_{\Sigma}$ is the identical morphism on $\Sigma=\{\mathrm{a}, \mathrm{b}, \mathrm{c}\}$, and $[f]$-primitive for the anti-/morphism $f$ with $f(\mathrm{a})=\mathrm{b}$, $f(\mathrm{~b})=\mathrm{a}$, and $f(\mathrm{c})=\mathrm{c}$. If $f$ is defined as a 3-cycle, e.g., $f(\mathrm{a})=\mathrm{c}, f(\mathrm{~b})=\mathrm{a}$, and $f(\mathrm{c})=\mathrm{b}$, then $w=\mathrm{a} f^{2}(\mathrm{a}) f(\mathrm{a})$ aa $f^{2}(\mathrm{a})$, so $w$ is an $[f]$-repetition both when $f$ acts as a morphism or an antimorphism. If $w=a b c c a b a b c$ and $f$ is defined such that $f(\mathrm{a})=\mathrm{b}, f(\mathrm{~b})=\mathrm{a}$, and $f(\mathrm{c})=\mathrm{c}$, we get that $w$ is and [ $f$ ]-repetition if $f$ acts as an antimorphism, and $w$ is $[f]$-primitive if $f$ acts as a morphism.

In the following, several classical repetition enforcing results are recalled. The first one is folklore (see, e.g., [3]). The next three are classical results of Fine and Wilf and Lyndon and Schützenberger, respectively.

Proposition 1 (1-in-2). A word $w \in \Sigma^{*}$ is a repetition if and only if $w$ occurs strictly inside $w w$. 
Theorem 1 (Fine \& Wilf [4]). Let $u, v \in \Sigma^{*}$. If two words $\alpha \in u\{u, v\}^{*}$ and $\beta \in$ $v\{u, v\}^{*}$ have a common prefix of length at least $|u|+|v|-d_{u, v}$, then $u$ and $v$ are powers of a common word of length $d_{u, v}$. The bound $|u|+|v|-d_{u, v}$ is optimal. some $i, j \geq 0$ and $p q$ is primitive.

Theorem 3 (Lyndon \& Schützenberger [5]). If $u^{\ell}=v^{m} w^{n}$ for some words $u, v, w \in$ $\Sigma^{*}$ and $\ell, m, n \geq 2$, then $u, v, w \in\{\gamma\}^{*}$ for some word $\gamma \in \Sigma^{*}$.

Theorem 4. Let $u, v \in \Sigma^{*}$ and $f: \Sigma^{*} \rightarrow \Sigma^{*}$ be a morphic permutation with $\operatorname{ord}(f)=$ $k+1$. Let $S(u, v)=\left\{u, f(u), \ldots, f^{k}(u), v, f(v), \ldots, f^{k}(v)\right\}^{*}$. If two words $\alpha \in u S(u, v)$ and $\beta \in v S(u, v)$ have a common prefix of length at least $|u|+|v|-d_{u, v}$, then there exists a word $\gamma \in V^{*}$, such that $u, v \in \gamma\left\{\gamma, f(\gamma), \ldots, f^{k}(\gamma)\right\}^{*}$.

Theorem 2 was extended in the setting of anti-/morphic involutions in [7]. Theorem 3 was extended for $[f]$-repetitions where $f$ is an antimorphic involution in a series of papers that culminated in [9], where a full characterisation of the triples $(\ell, m, n)$ for which the equation $u_{1} u_{2} \cdots u_{\ell}=v_{1} v_{2} \cdots v_{m} w_{1} w_{2} \cdots w_{n}$, where $u_{i} \in\{u, f(u)\}$ for all $i \in[\ell], v_{j} \in\{v, f(v)\}$ for all $j \in[m]$, and $w_{k} \in\{w, f(w)\}$ for all $k \in[n]$, has only solutions which are $[f]$-repetitions was given.

\section{The Morphic Case}

In this section some well known equations which only have repetitions as solutions are generalised to equations whose solutions are repetitions under morphic permutations. These results are used to ultimately show that a version of Theorem 3 holds for $[f]$-repetitions in the case that $f$ is a morphic permutation.

\subsection{Preliminary results}

Some basic lemmas are first established, which provide some fundamental combinatorial tools for proving the later results. They focus on two very well-known equations, namely $x y=y x$ and $x y=y z$ with $x, y, z \in \Sigma^{+}$, and describe their solutions in this more general setting.

Lemma 1. Let $x, y \in \Sigma^{+}, f$ a morphic permutation on $\Sigma^{*}$, and $a, b, c, d \in[\operatorname{ord}(f)]_{0}$ with

$$
f^{a}(x) f^{b}(y)=f^{c}(y) f^{d}(x) .
$$

Then there exists a word $\gamma \in \Sigma^{+}$such that $x, y \in\left\{\gamma, f(\gamma), \ldots, f^{\operatorname{ord}(f)-1}(\gamma)\right\}^{*}$.

Proof. Theorem 4 can be applied for $\alpha=f^{a}(x) f^{b}(y), \beta=f^{c}(y) f^{d}(x), u=f^{a}(x)$, and $v=f^{c}(y)$. Clearly, $\alpha$ and $\beta$ have a common prefix of length $|\alpha|=|\beta|=|u|+|v|$, it follows that there exists $\gamma$ such that $f^{a}(x), f^{c}(y) \in \gamma\left\{\gamma, f(\gamma), \ldots, f^{\text {ord }(f)-1}(\gamma)\right\}^{*}$. The conclusion follows. 
Consider for instance $\Sigma=\{0,1,2,3\}$ and $f: \Sigma \rightarrow \Sigma$ with $f(0)=1, f(1)=3$, $f(2)=0$, and $f(3)=2$. Then we have $f^{2}(32) f(20)=0101=f(20) f^{2}(32)$ and $32=f^{2}(01)$ and $20=f^{3}(01)$ which leads to the common root 01 .

While Lemma 1 provides a direct analogy to the standard setting, for which the "repetition-enforcing" nature of the equation is folklore, it is also possible to provide the following more specific insight which is essential to the proofs.

Lemma 2. Let $x, y \in \Sigma^{+}$with $x=x_{1} x_{2}$ such that $\left|x_{1}\right|=\left|x_{2}\right|$, $f$ a morphic permutation on $\Sigma^{*}$, and $a, b, c \in[\operatorname{ord}(f)-1]_{0}$ with

$$
y x_{1} x_{2}=f^{a}\left(x_{1}\right) f^{b}\left(x_{2}\right) f^{c}(y)
$$

Then there exists $\gamma \in \Sigma^{+}$such that $x, y, f^{a}\left(x_{1}\right) f^{b}\left(x_{2}\right)$ are in $\left\{\gamma, f(\gamma), \ldots, f^{\operatorname{ord}(f)-1}(\gamma)\right\}^{*}$.

PROOF. The statement is shown by induction on $|x|$.

If $|x|=2$, then there are two cases to be analysed: $|y|=2 k$ or $|y|=2 k+1$. In the first case, we have $y=f^{-k c}(x) \cdots f^{-2 c}(x) f^{-c}(x)$ and the conclusion follows for $\gamma=x$. The case $|y|=2 k+1$ implies $y=f^{-c}\left(x_{2}\right)$ if $k$ is zero and

$$
y=f^{-(k+1) c}\left(x_{2}\right) f^{-k c}(x) \cdots f^{-2 c}(x) f^{-c}(x)
$$

otherwise. Hence, $x_{1}=f^{-(k+1) c-a}\left(x_{2}\right)$, and $x, y, f^{a}\left(x_{1}\right) f^{b}\left(x_{2}\right)$ are $[f]$-repetitions of root $x_{2}$.

Let us assume that the claim holds for $|x|<2 n$. We will show that it also holds for $|x|=2 n$.

If $y x_{1} x_{2}=f^{a}\left(x_{1}\right) f^{b}\left(x_{2}\right) f^{c}(y)$ is true with $|y|=\left|x_{1}\right|$, then the conclusion follows immediately, since $x, f^{a}\left(x_{1}\right) f^{b}\left(x_{2}\right)$ and $y$ are $[f]$-repetitions of the root $x_{1}$. If $|y|<\left|x_{1}\right|$, then there exist $i_{1}, \ldots, i_{k}$ and $j_{1}, \ldots, j_{k}$ and $v_{1}^{\prime}, v_{2}^{\prime} \in \Sigma^{*}$ such that $x_{1}=$ $f^{i_{1}}(y) \ldots f^{i_{k}}(y) v_{1}^{\prime}$ and $x_{2}=v_{2}^{\prime} f^{j_{k}}(y) \ldots f^{j_{1}}(y)$ hold with $\left|v_{1}^{\prime}\right|=\left|v_{2}^{\prime}\right|<|y|$. The conclusions follow immediately if $v_{1}^{\prime}$ is the empty word. Otherwise, it follows that

$$
f^{i_{k}}(y) v_{1}^{\prime} v_{2}^{\prime}=f^{a}\left(v_{1}^{\prime}\right) f^{b}\left(v_{2}^{\prime}\right) f^{j_{k}+c}(y)
$$

holds. By the induction hypothesis, the existence of $\gamma \in \Sigma^{*}$ is implied such that $v_{1}^{\prime} v_{2}^{\prime}, f^{a}\left(v_{1}^{\prime}\right) f^{b}\left(v_{2}^{\prime}\right)$, and $y$ are $[f]$-repetitions of root $\gamma$. The statement follows.

If $|x|>|y|>\left|x_{1}\right|$, choose $\alpha \neq \epsilon$ as the prefix of $x_{1} x_{2}$ such that $\alpha f^{c}(y)=x_{1} x_{2}$. Then $f^{-b}(\alpha)$ is a suffix of $x_{2}$. Thus $x_{1}=\alpha v_{1}$ and $x_{2}=v_{2} f^{-b}(\alpha)$. It follows that $f^{c}(y)=v_{1} v_{2} f^{-b}(\alpha)$ and $y=f^{-a}(\alpha) f^{-a}\left(v_{1}\right) f^{-b}\left(v_{2}\right)$ hold. The combination of both yield

$$
f^{-c}\left(v_{1}\right) f^{-c}\left(v_{2}\right) f^{-b-c}(\alpha)=f^{-a}(\alpha) f^{-a}\left(v_{1}\right) f^{-b}\left(v_{2}\right) .
$$

By the induction hypothesis, there exists $\gamma$ such that $f^{-c}\left(v_{1}\right) f^{-c}\left(v_{2}\right)$ and $\alpha$ are $[f]-$ repetitions of root $\gamma$. It follows that $x, y, f^{a}\left(x_{1}\right) f^{b}\left(x_{2}\right)$ are also $[f]$-repetitions of root $\gamma$.

Finally, if $|y|=|x|$, the conclusion is immediate. If $|y|>|x|$, then there exist $k$ such that $y=f^{-(k+1) c}\left(y^{\prime}\right) f^{-k c}(x) \cdots f^{-2 c}(x) f^{-c}(x)$ and $y^{\prime}$ a suffix of $x$. It follows that

$$
f^{-(k+1) c}\left(y^{\prime}\right) f^{-k c}(x)=f^{a}\left(x_{1}\right) f^{b}\left(x_{2}\right) f^{k c}\left(y^{\prime}\right),
$$

so the same reasoning as in the previous case applies. The conclusion follows once more, and, with this case, the statement is completely proven. 
Lemma 3. Let $x, y, z \in \Sigma^{+}, f$ a morphic permutation on $\Sigma^{*}$ and $a, b, c, d \in[\operatorname{ord}(f)-$ $1]_{0}$. If the equality

$$
f^{a}(x) f^{b}(y)=f^{c}(y) f^{d}(z)
$$

holds then there exist $u, v \in \Sigma^{*}, i, s, r, q \in \mathbb{N}_{0}$ with

$$
x=u v, \quad z=f^{q}(v) f^{q+r}(u), \quad \text { and } \quad y=f^{s+r}(u v) \ldots f^{s+i r}(u v) f^{s+(i+1) r}(u) .
$$

Proof. Consider first the case $|x| \geq|y|$. Choose $u, v \in \Sigma^{*}$ with $x=u v$ and $|u|=|y|$ and thus $|u|=\left|f^{c}(y)\right|$. This implies $f^{a}(x)=f^{a}(u) f^{a}(v)$ and thus $f^{a}(u)=f^{c}(y)$. The latter one implies $f^{b}(y)=f^{b+a-c}(u)$ and, with $f^{d}(z)=f^{a}(v) f^{b}(y)$, we get that

$$
z=f^{a-d}(v) f^{b-d}(y)=f^{a-d}(v) f^{b+a-d-c}(u)
$$

holds. Thus the lemma holds for $q=a-d, r=b-c, s=a-b$ and $i=0$ (according to the discussion in Section $2 f^{e}$ is well defined for $\left.e \in\{q, r, s, i\}\right)$. On the other hand in the case $|x|<|y|$, there exists $v^{\prime} \in \Sigma^{+}$with $f^{c}(y)=f^{a}(x) v^{\prime}$ and $f^{b}(y)=v^{\prime} f^{d}(z)$. This implies

$$
f^{a-c}(x) f^{-c}\left(v^{\prime}\right)=y=f^{-b}\left(v^{\prime}\right) f^{d-b}(z) .
$$

From $\left|v^{\prime}\right|<|y|$ and by an inductive argument, there exists $u, v \in \Sigma^{*}$ with $x=u v$ and $z=f^{r_{1}}(v) f^{r_{2}}(u)$ for $r_{1}, r_{2} \in[\operatorname{ord}(f)]_{0}$. Moreover there exist $g_{1}, \ldots, g_{i+1} \in[\operatorname{ord}(f)]_{0}$ for some $i \in \mathbb{N}_{0}$ with $v^{\prime}=f^{g_{1}}(u v) \ldots f^{g_{i}}(u v) f^{g_{i+1}}(u)$. This implies

$$
y=f^{a-c}(u v) f^{g_{1}-c}(u v) \ldots f^{g_{i}-c}(u v) f^{g_{i+1}-c}(u) .
$$

By plugging these values for $x, y$ and $z$ into the original equation, it is clear that the equation is satisfied exactly when $g_{1}=b+a-c, g_{j+1}=g_{j}+b-c$ (so that $g_{j}=$ $a+j(b-c)), r_{1}+d=g_{i}+b-c$ and $r_{2}+d=g_{i+1}+b-c=r_{1}+(b-c)$. Choosing $r=b-c, s=a-b$ and $q=r_{1}$ shows that the statement of the lemma is true.

If three words $x, y, z$ satisfy the equation from Theorem 2 (i.e., $x y=y z$ ) then the words $x$ and $z$ are called conjugate, denoted $x \sim z$ for short. It is well known that the conjugacy relation is an equivalence relation. When working in the setting of equations under morphic permutations, this relation can be extended to $f$-conjugacy. For a morphic permutation $f$, the words $x, y \in \Sigma^{*}$ are said to be $f$-conjugate (written $\left.x \sim_{f} y\right)$ if there exist $a, b, c, d \in[\operatorname{ord}(f)]_{0}$ such that $f^{a}(x) f^{b}(y)=f^{c}(y) f^{d}(z)-$ so if they satisfy the equality addressed in Lemma 3 It can be seen that $x \sim_{f} y$ follows from $x \sim y$. More interestingly however, while $\sim_{f}$ is symmetrical and reflexive, it is not 170 transitive (unless $f$ is the identical morphism). To see why $\sim_{f}$ is not an equivalence, assume that $f$ is not the identical morphism. Thus, there exists $a \in \Sigma$ such that $f(a)=$ $b \neq a$. Then $a a a \sim_{f} b a a$ (because, $\left.f(a a a) a a=f(a a) f^{0}(b a a)\right)$ and $b a a \sim_{f} a b a$ (because, $b a a \sim a b a$ ). However, $a a a \nsim_{f} a b a$. Indeed, if $a a a \sim_{f} w$, then $|w|=3$ and $w=f^{q}(v) f^{q+r}(u)$ for some $q, r \in \mathbb{N}_{0}$ and $u, v$ such that $a a a=u v$. It is immediate that $a b a$ is not such a word $w$. Accordingly, $\sim_{f}$ is an equivalence if and only if $f$ is the identity morphism.

The following lemma extends another fundamental result mentioned in Proposition 1 , and will be proven by reducing to the case considered by Lemma11(see Fig. 1). 
Lemma 4. Let $f: \Sigma^{*} \rightarrow \Sigma^{*}$ be a morphic permutation and $a, b, c \in[\operatorname{ord}(f)-1]_{0}$. If $u \in \Sigma^{*}$ is $[f]$-primitive, then for $x, y \in \Sigma^{*}$ with

$$
f^{a}(u) f^{b}(u)=x f^{c}(u) y
$$

either $x=\varepsilon$ or $y=\varepsilon$ follows.

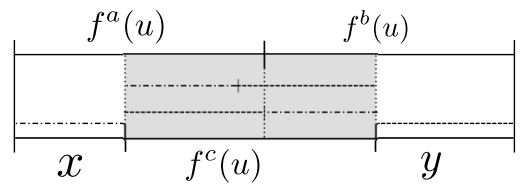

Figure 1: $x, y$ reoccur each twice within $f^{c}(y)$, such that - except for permutation application - the pattern $x y=y x$ occurs (shown by the dotted and dashed lines), so Lemma 1 may be applied.

PROOF. Suppose that neither $x$ nor $y$ are empty. Since $x$ is a prefix of $f^{a}(u), f^{c}(u)$ starts with $f^{c-a}(x)$, and analogously $f^{c}(u)$ ends in $f^{c-b}(y)$ since $f^{b}(u)$ ends in $y$. With $|u|=|x|+|y|$ this implies $f^{c}(u)=f^{c-a}(x) f^{c-b}(y)$. Applying the same arguments to $f^{b}(u)$ and $f^{a}(u)$ leads to $f^{a-b}(y) \in \operatorname{Pref}\left(f^{c}(u)\right)$ and $f^{b-a}(x) \in \operatorname{Suff}\left(f^{c}(u)\right)$, i.e. $f^{c}(u)=f^{a-b}(y) f^{b-a}(x)$. This implies

$$
f^{c-a}(x) f^{c-b}(y)=f^{a-b}(y) f^{b-a}(x)
$$

and by Lemma 1$]$ is not $[f]$-primitive.

Lemma2 can be extended in a similar fashion.

Lemma 5. Let $f: \Sigma^{*} \rightarrow \Sigma^{*}$ be a morphic permutation and $a, b, c, d \in[\operatorname{ord}(f)-1]_{0}$. If $u \in \Sigma^{*}$ is $[f]$-primitive and $u=u_{1} u_{2}$, with $\left|u_{1}\right|=\left|u_{2}\right|$, then for $x, y \in \Sigma^{*}$ with

$$
f^{a}(u) f^{b}(u)=x f^{c}\left(u_{1}\right) f^{d}\left(u_{2}\right) y
$$

either $x=\varepsilon$ or $y=\varepsilon$ follows.

\subsection{A generalisation of Theorem 3}

In the rest of this section it will be shown that Lyndon and Schützenberger's result can be generalised without any additional restrictions in the setting of repetitions under morphic permutations. In this setting, the Lyndon \& Schüzenberger equation is defined for the word-variables $u, v, w$ (over $\Sigma^{+}$) by

$$
f^{a_{1}}(u) \ldots f^{a_{r}}(u) f^{c_{1}}(v) \ldots f^{c_{s}}(v)=f^{b_{1}}(w) \ldots f^{b_{t}}(w),
$$

and parametrised by the integers $r, s, t \in \mathbb{N}_{\geq 2}, a_{i}, b_{k}, c_{j} \in[\operatorname{ord}(f)]_{0}, i \in[r], k \in[s]$, $185 j \in[t]$, and a morphic permutation $f$ on $\Sigma^{*}$.

For simplicity, the following notations are sometimes used: $\alpha_{1}=f^{a_{1}}(u) \ldots f^{a_{r}}(u)$, $\alpha_{2}=f^{c_{1}}(v) \ldots f^{c_{s}}(v)$, and $\beta=f^{b_{1}}(w) \ldots f^{b_{t}}(w)$.

The intention is to show that there exists a word $\gamma$ such that the solutions of Equation 1 can be expressed as $u, v, w \in\left\{\gamma, f(\gamma), \ldots, f^{\text {ord }(f)-1}(\gamma)\right\}^{*}$. Hence, we show that the 
generalisation of the Lyndon and Schützenberger's equation, when augmented by the presence of morphic permutations, remains repetition enforcing.

In order to show that indeed $u, v$, and $w$ are $[f]$-repetitions with the same root under these conditions, the proof is divided into various cases. To begin with, the cases in which the equation has some solutions $u, v, w$ such that $u$ s and $v$ s "fit" exactly inside the $w$ s and vice-versa are given.

Lemma 6. If the words $u, v, w$ satisfy Equation 1 , with $r, s, t \geq 2$, and $|u|$ divides $|w|$ or, alternatively, $|v|$ divides $|w|$, then there exists a word $v$ for which we have that $u, v, w \in\left\{v, f(v), \ldots, f^{\text {ord }(f)-1}(v)\right\}^{*}$.

PRoof. Consider w.l.o.g. $|u|$ divides $|w|$, i.e. $d_{u, w}=|u|$.

case 1: $\left|\alpha_{1}\right|<|u|+|w|-d_{u, w}$

With $r \geq 2$ we have $\left|\alpha_{1}\right| \geq 2|u|$ and by $|w|>\left|\alpha_{1}\right|$ we get $|w| \geq 2|u|$. Consequently $\left|\alpha_{2}\right|>|w| \geq 2|u|$. On the other hand, by $s \geq 2$, it follows that $\left|\alpha_{2}\right| \geq 2|v|$. This results in

$$
\left|\alpha_{2}\right| \geq|u|+|v|>|u|+|v|-d_{u, v},
$$

and Fine \& Wilf's Theorem is applicable on $\alpha_{2}$ and the prefix of $\beta$ of length $r|u|+|v|>$ $|u|+|v|-d_{u, v}$, i.e. $u$ and $v$ are $[f]$-repetitions. Since $|u|$ divides $|w|, w$ is an $[f]$ repetition as well.

case 2: $\left|\alpha_{1}\right| \geq|u|+|w|-d_{u, w}$

In this case the Theorem of Fine and Wilf is directly applicable and provides a $v \in \Sigma^{*}$ such that $u, w$ are $[f, v]$-repetitions.

case 2.a: $|\gamma| \leq|v|$ If $|\gamma|$ divides $|v|$, then $v$ is also a $[f, \gamma]$-repetition. Consider

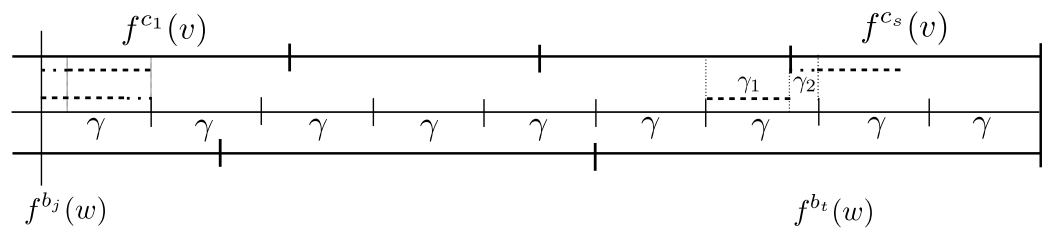

Figure 2: The factors building the permutation of $\gamma$ at the point to the last permutation of $v$ occur in the first permutation of $v$ in the pattern $\gamma_{1} \gamma_{2}=\gamma_{2} \gamma_{1}$ - neglecting the permutations

$|\gamma|+|v|$. Then the point $\left|\alpha_{1}\right|+(s-1)|v|$ is in an $f$-permutation of $\gamma$, i.e. there exist $k_{1} \in \mathbb{N}$ and $\gamma_{1}, \gamma_{2} \in \Sigma^{*}$ with $f^{k_{1}}(\gamma)=\gamma_{1} \gamma_{2}$ such that $\gamma_{1}$ is a suffix of $f^{c_{s-1}}(v)$, and $\gamma_{2}$ is a prefix of $f^{c_{s}}(v)$. Since $\alpha_{2}$ and in particular $f^{c_{s}}(v)$ ends with a permutation of $\gamma$ there exists a $k_{2} \in \mathbb{N}$ such that $\gamma_{2} f^{k_{2}-k_{1}}\left(\gamma_{1}\right)$ is a prefix of $f^{c_{s}}(v)$. This implies that $f^{c_{1}-c_{s}}\left(\gamma_{2}\right) f^{k_{2}-k_{1}+c_{1}-c_{2}}\left(\gamma_{1}\right)$ is a prefix of $f^{c_{1}}(v)$. Since $\alpha_{1}$ ends with a permutation of $\gamma$, $\alpha_{2}$ has a permutation $f^{k_{3}}(\gamma)$ as a prefix for a $k_{3} \in \mathbb{N}$. This results in

$$
f^{c_{1}-c_{s}}\left(\gamma_{2}\right) f^{k_{2}-k_{1}+c_{1}-c_{2}}\left(\gamma_{1}\right)=f^{k_{3}}\left(\gamma_{1}\right) f^{k_{3}}\left(\gamma_{2}\right) .
$$

Lemma 1 yields a $\delta \in \Sigma^{*}$ such that $\gamma_{1}, \gamma_{2}$ are $[f, \delta]$-repetitions. Since $u, v$, and $w$ are concatenations of $\gamma_{1}$ and $\gamma_{2}$, they are $[f, \delta]$-repetitions as well. We can take $v=\delta$. 
case 2.b: $|\gamma|>|v|$

If $|v|$ divides $|\gamma|$ then $u, v, w$ are $[f, v]$-repetitions. Consider $|v|+|\gamma|$. This and using $|v|<|\gamma|$ implies $\left|\alpha_{2}\right| \geq 2|\gamma|$. Thus, there exists a $k \in \mathbb{N}_{\geq 2}$ with

$$
\left|\alpha_{2}\right| \geq k|\gamma|=(k-1)|\gamma|+|\gamma|>|\gamma|+|v|>|\gamma|+|v|-d_{\gamma, v} .
$$

Again by Fine and Wilf's Theorem $\gamma$ and $v$ are $[f, \delta]$-repetitions for a $\delta \in \Sigma^{*}$ and since $u$ and $w$ are $[f]$-repetitions of $\gamma$, they are also $[f]$-repetitions of $\delta$. Again, we can take $v=\delta$

Lemma 7. If $u, v, w$ are words that satisfy Equation 1$]$ with $r, s, t \geq 2$, and $|w|$ divides $|u|$ or, alternatively, $|w|$ divides $|v|$, then there exists a word $\gamma \in \Sigma^{*}$ such that $u, v, w \in$ $\left\{\gamma, f(\gamma), \ldots, f^{\operatorname{ord}(f)-1}(\gamma)\right\}^{*}$.

Proof. Consider w.l.o.g. $|w|$ divides $|u|$. Then $\alpha_{1}$ is a repetition of $w$ and consequently $\alpha_{2}$ as well. If $\alpha_{2}$ contains only one $w$, then $w$ is an $[f, v]$-repetition which propagates to $u$. If $\alpha_{2}$ contains more than one $w$, then $\left|\alpha_{2}\right| \geq 2|v|$ and $\left|\alpha_{2}\right| \geq 2|w|$ hold and this implies

$$
\left|\alpha_{2}\right| \geq|v|+|w|>|v|+|w|-d_{v, w} .
$$

By Fine and Wilf's Theorem we get that $w, v$ are $[f]$-repetitions and, since $|w|$ divides $|u|$, then $u$ is a $[f]$-repetition as well.

The following lemma demonstrates how, in some cases, the extension of the Theorem of Fine and Wilf (Theorem 4), may be applied. This is straightforward if the theorem may be applied from both endpoints, in opposite directions, showing first that $u$ and $w$ share an $f$-root and then that $v$ and $w$ share an $f$-root. In fact, as the lemma states, it is sufficient to be able to apply the theorem in just one direction - although this requires more effort to prove.

Lemma 8. If $u, v, w$ are words satisfying Equation 1 such that $\alpha_{1}$ and $\beta$ have a common prefix of length at least $|w|+|u|-d_{u, w}$ and $\alpha_{2}$ and $\beta$ have common suffix of length at least $|w|+|v|-d_{v, w}$, then there exists a word $\gamma$ such that $u, v, w \in\left\{\gamma, f(\gamma), \ldots, f^{\operatorname{ord}(f)-1}(\gamma)\right\}^{*}$.

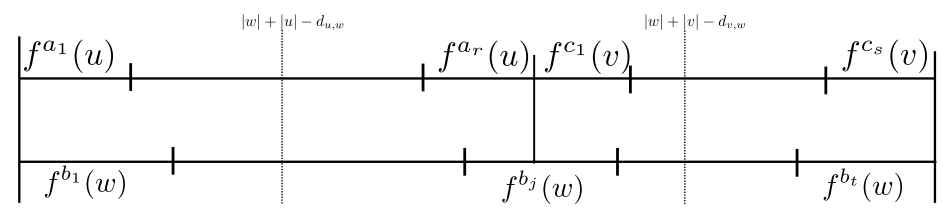

Figure 3: If the parts of permutations of $u$ and the one permutation of $v$ are each long enough, Theorem 4 can be applied from either side.

PROOF. By Theorem 4 there exist $\gamma_{1}, \gamma_{2} \in \Sigma^{*}$ such that $u, w$ are $\left[f, \gamma_{1}\right]$ - and $u, v$ are $\left[f, \gamma_{2}\right]$-repetitions. Applying the Theorem again seperately to $w$ as an $[f]$-repetition of $\gamma_{1}$ and as one of $\gamma_{2}$. This implies that $\gamma_{1}$ and $\gamma_{2}$ are $[f, \gamma]$-repetitions for a $\gamma \in \Sigma^{*}$. The application is possible, because not only $u$ but also $v$ are non-empty and consequently 
$\beta$ has at least the length $|u|+|v| \geq\left|\gamma_{1}\right|+\left|\gamma_{2}\right|>\left|\gamma_{1}\right|+\left|\gamma_{2}\right|-\operatorname{gcd}\left(\left|\gamma_{1}\right|,\left|\gamma_{2}\right|\right)$. Since $u$ and $v$ are $[f]$-repetitions of $\gamma_{1}$ and $\gamma_{2}$, respectively, $u$ and $v$ are also [f]-repetitions of $\gamma$.

For completeness, the proof that one of the length constraints is sufficient is given.

Lemma 9. It is sufficient in Lemma 8 if either the condition for the prefix or the condition for the suffix holds.

Proof. Note that in Equation 1, we have $r, s, t \geq 2$. Now, assume w.l.o.g. that

$$
\left|\alpha_{1}\right| \geq|u|+|w|-d_{u, w} \text { and }\left|\alpha_{2}\right|<|v|-|w|-d_{v, w}
$$

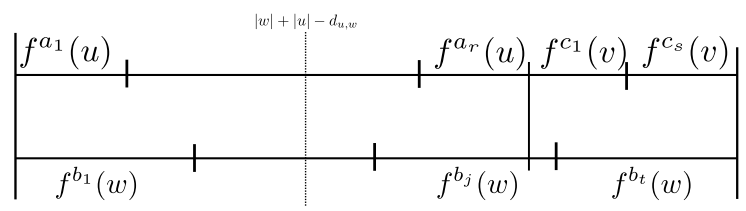

Figure 4: In contrast to Fig. 3here only the common prefix of the permutations of $u$ and $w$ is long enough to apply Fine \& Wilf's theorem.

By the Theorem of Fine and Wilf exists a $\gamma \in \Sigma^{*}$ such that $u, w$ are $[f, \gamma]$-repetitions. Consider w.l.o.g. $|\gamma|=d_{u, w}$. We further split the discussion into cases, depending on the value of $s$, the number of images $f^{c_{i}}(v)$ of the word $v$ under iterations of $f$. The word

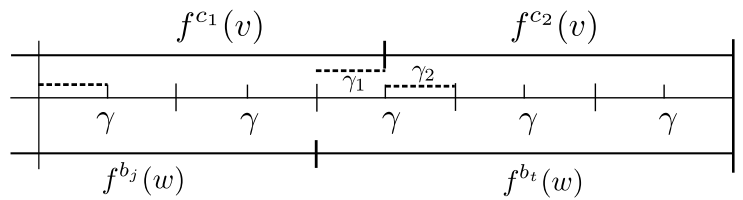

Figure 5: In the case of two permutations of $v$ the root $\gamma$ can be split into two factors which are permutations of each other.

$\alpha_{2}$ starts with a permutation of $\gamma$. If $|\gamma|=\left|\alpha_{2}\right|$ then $u, v$, and $w$ are $[f, v]$-repetitions. If $\gamma$ is a proper prefix of $\alpha_{2}$, we have $|\gamma| \leq \frac{\left|\alpha_{2}\right|}{2}$ since $|\gamma|$ divides $|w|$. Moreover by $|v| \leq \frac{\left|\alpha_{2}\right|}{2}$, we get $|\gamma|+|v| \leq\left|\alpha_{2}\right|$. Applying Theorem 4 to $\alpha_{2}$ we get the conclusion.

Following from Lemma 8 it is now possible to show that Equation 1 is repetition 235 enforcing provided $r, s$ and $t$ are large enough.

Proposition 2. If $u, v, w$ are words satisfying Equation 1, with $r, s, t$ at least 3 or with $t \geq 4$ and $r$, s at least 2, then one of the conditions of Lemma 8 also holds. Hence, there exists a word $\gamma$ such that $u, v, w \in\left\{\gamma, f(\gamma), \ldots, f^{\operatorname{ord}(f)-1}(\gamma)\right\}^{*}$. 
PROOF. In the case when $t \geq 4$, it is immediate that at least one of $\alpha_{1}$ or $\alpha_{2}$ should be greater than 2|w|. Assume, w.l.o.g., $\alpha_{1} \geq 2|w|$. Also, $\alpha_{1} \geq 2|u|$ (as $r \geq 2$ ) so the conditions of Lemma 8 are fulfilled. Therefore, this lemma can be applied to get the conclusion.

The other case is discussed in the following. Suppose that $\left|\alpha_{1}\right|<|w|+|u|-d_{u, w} \wedge$ $\left|\alpha_{2}\right|<|w|+|v|-d_{v, w}$. If $|u|$ and $|v|$ aren't greater than $\frac{1}{2}|w|$, then

$$
\begin{aligned}
|\beta| & =\left|\alpha_{1}\right|+\left|\alpha_{2}\right|<2|w|+|u|+|v|-d_{u, w}-d_{v, w} \\
& \leq 2|w|+\frac{1}{2}|w|+\frac{1}{2}|w|-d_{u, w}-d_{v, w} \\
& =3|w|-d_{u, w}-d_{v, w}
\end{aligned}
$$

holds which is a contradiction to $t \geq 3$. On the other hand if $|u|>\frac{1}{2}|w|$ would hold,

$$
\left|\alpha_{1}\right| \geq 3|u|=|u|+2|u|>|u|+|w|>|u|+|w|-d_{u, w}
$$

would follow.

Recalling the original Lyndon-Schützenberger result (Theorem 3), the claim we made in the beginning of this subsection is nearly proven for the permutation setting as well. It remains to prove that the same result holds when $r, s, t$ are all equal to 2 , or when $t=3$ and at least one of $r$ or $s$ is 2 , respectively.

Let us first reformulate the result of Lemma 3 in a way that will be used here.

Remark 1. Let $w_{1}, w_{2}$, be in $\Sigma^{+}, f$ a permutation on $\Sigma$, and $a, b, c, d \in \mathbb{N}$. If

$$
f^{a}\left(w_{2}\right) \text { is a suffix of } f^{c}\left(w_{2}\right) f^{d}(x),
$$

then there exists a suffix $x^{\prime}$ of $f^{n_{0}}(x)$, for some $n_{0} \in \mathbb{N}$, and there exists $n_{1}, \ldots, n_{r} \in \mathbb{N}$ for $r \in \mathbb{N}$ with

$$
w_{2}=x^{\prime} f^{n_{1}}(x) \ldots f^{n_{r}}(x) .
$$

If $f^{a}\left(w_{1}\right)$ is a prefix of $f^{b}(x) f^{c}\left(w_{1}\right)$, an analogous result can be obtained.

In the following, we address first the case when $t=2$. We begin with the case when also $r=s=2$, which is rather straightforward (Lemma 10). Next, we address the case $s=2$ and $r \geq 3$ in Lemma 11, note that this case is symmetric to the case when $r=2$ and $s \geq 3$, so the respective case follows too. Finally, we present the case when $r$ and $s$ are both at least 3, in Lemma 12 There are two cases left for $t=3$. The first one is 255 when $r=s=2$. The second one is when $s=3$ and $r=2$ (and its symmetrical case $r=3$ and $s=2$, which is analoguous). They are treated in Lemmas 13 and 14

Lemma 10. If $u, v, w$ are words satisfying Equation 1] with $r=s=t=2$, then there exists a word $t$ such that $u, v, w \in\left\{\gamma, f(\gamma), \ldots, f^{\operatorname{ord}(f)-1}(\gamma)\right\}^{*}$. 


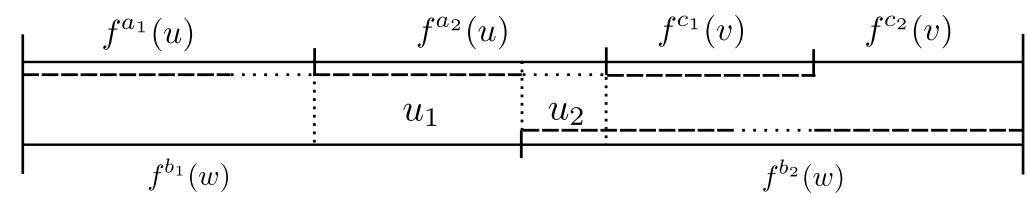

Figure 6: In the case of $r=s=t=2$ the pattern $u_{1} u_{2}=u_{2} u_{1}$ - neglecting the permutations - occurs in the second $w$.

Proof. Consider w.l.o.g. $2|u|>|w|$. Choose $u_{1}, u_{2} \in \Sigma^{+}$with $f^{a_{2}}(u)=u_{1} u_{2}$ such that $u_{1} \in \operatorname{Suff}\left(f^{b_{1}}(w)\right)$ and $u_{2} \in \operatorname{Pref}\left(f^{b_{2}}(w)\right)$ (see Figure 6). Hence, $f^{b_{1}}(w)=$ $f^{a_{1}-a_{2}}\left(u_{1}\right) f^{a_{1}-a_{2}}\left(u_{2}\right) u_{1}$ and

$$
f^{b_{2}-b_{1}+a_{1}-a_{2}}\left(u_{1}\right) f^{b_{2}-b_{1}+a_{1}-a_{2}}\left(u_{2}\right) f^{b_{2}-b_{1}}\left(u_{1}\right)=f^{b_{2}}(w)=u_{2} f^{c_{1}}(v) f^{c_{2}}(v) .
$$

By this, it follows that $2\left|u_{1}\right|=2|v|$. Moreover, since $f^{b_{2}-b_{1}}\left(u_{1}\right)$ and $f^{c_{2}}(v)$ are suffixes of $f^{b_{2}}(w)$, we get $f^{b_{2}-b_{1}}\left(u_{1}\right)=f^{c_{2}}(v)$. Substituting this result in $f^{b_{2}}(w)$ leads to

$$
f^{b_{2}-b_{1}+a_{1}-a_{2}}\left(u_{1}\right) f^{b_{2}-b_{1}+a_{1}-a_{2}}\left(u_{2}\right)=u_{2} f^{c_{1}-c_{2}+b_{2}-b_{1}}\left(u_{1}\right) .
$$

By Lemma1 1 we get the existence of a $\gamma \in \Sigma^{*}$ such that $u_{1}, u_{2}$ are $[f, \gamma]$-repetitions and consequently $u, v$, and $w$ are $[f]$-repetitions as well.

Lemma 11. If $u, v, w$ are words satisfying Equation 1 for $s=t=2$ and $r \geq 3$, then there exists a word $v$ such that $u, v, w \in\left\{v, f(v), \ldots, f^{\operatorname{ord}(f)-1}(v)\right\}^{*}$.

PROOF. We will perform a case analysis.

case 1: Consider first $r|u|<|w|$ and choose $x \in \Sigma^{*}$ with $f^{b_{1}}(w)=\alpha_{1} x$. Then $f^{b_{2}-b_{1}}(x)$ is suffix of $f^{b_{2}}(w)$ and $f^{c_{2}}(v)$. This implies

$$
f^{c_{1}}(v) f^{c_{2}}(v)=x f^{b_{2}-b_{1}}\left(\alpha_{1}\right) f^{b_{2}-b_{1}}(x) .
$$

Hence the point $2|w|-|v|$ is in the middle of $f^{b_{2}-b_{1}}\left(\alpha_{1}\right)$. Choose $x^{\prime}, x^{\prime \prime} \in \Sigma^{+}$with $f^{c_{1}}(v)=x x^{\prime}$ and $f^{c_{2}}(v)=x^{\prime \prime} f^{b_{2}-b_{1}}(x)$.

If $r$ is even, then $f^{c_{1}}(v)=x f^{a_{1}-b_{1}+b_{2}}(u) f^{a_{2}-b_{1}+b_{2}}(u) \cdots f^{a_{r / 2}-b_{1}+b_{2}}(u)$ and $f^{c_{2}}(v)=$ $f^{a_{r / 2+1}-b_{1}+b_{2}}(u) \cdots f^{a_{r}-b_{1}+b_{2}}(u) f^{b_{2}-b_{1}}(x)$. As $r \geq 4$, we get that $f^{c_{1}}(v) \in x S(x, u)$ and $f^{c_{2}}(v) \in f^{a_{r / 2+1}-b_{1}+b_{2}}(u) S(x, u)$, and $|v| \geq|v|+|u|$. As such, the conclusion follows now directly via Theorem 1 .

Assume $r$ now to be odd. In this case, the point $2|w|-|v|$ is in the middle of a permutation of $u$ and thus there exist $y, y^{\prime} \in \Sigma^{+}$with $f^{\frac{a r}{2}+1-b_{1}+b_{2}}(u)=y y^{\prime},|y|=\left|y^{\prime}\right|$, $y \in \operatorname{Suff}\left(f^{c_{1}}(v)\right)$, and $y^{\prime} \in \operatorname{Pref} f^{c_{2}}(v)$. Before this factorisation can be used again, it is necessary to have a look at the occurrences of $x$ 's permutations. Since $f^{b_{2}-b_{1}}(x)$ is a suffix of $f^{c_{2}}(v), f^{b_{2}-b_{1}-c_{2}+c_{1}}(x)$ is a suffix of $f^{c_{1}}(v)$.

This means that $f^{b_{2}-b_{1}-c_{2}+c_{1}}(x)$ and $x^{\prime}$ have a common suffix. If they are of equal length, $x$ is on the one hand an alternation of permutations of $y^{\prime}$ and $y$ (induced by $x^{\prime \prime}$ ) and on the other hand an alternation of permutations of $y$ and $y^{\prime}$ (induced by $x^{\prime}$ ). From 
this we obtain the fact that $y$ and $y^{\prime}$ are permutations of each other because they have the same length. Moreover $u, v$, and $w$ are built up by permutations of $y$.

Assume for the second case that $|x|>\left|x^{\prime}\right|$. With the same transfers from $x$ resp. $x^{\prime}$ and the fact that the $y^{\prime}-y$-alternation is followed by a $\gamma_{1} \in \Sigma^{+}$whose image under an iteration of $f$ occurs before $x^{\prime}$ in $f^{b_{1}}(w)$, we get

$$
f^{\ell_{1}}\left(y^{\prime}\right) f^{\ell_{2}}(y) \ldots f^{\ell_{n}}\left(y^{\prime}\right) f^{\ell_{n+1}}\left(\gamma_{1}\right)=\gamma_{1} f^{k_{1}}(y) f^{k_{1}}\left(y^{\prime}\right) \ldots f^{k_{n}}(y),
$$

with exponents in $[\operatorname{ord}(f)]_{0}$ (renamed for convenience). By $\left|\gamma_{1}\right|<\left|y^{\prime}\right|, f^{\ell_{1}}\left(y^{\prime}\right)$ and $f^{k_{1}}(y)$ overlap, let $\gamma_{2} \in \Sigma^{+}$be this overlap. This implies that $\gamma_{2}$ and $\gamma_{1}$ are permutations of each other and hence such a permutation is a prefix of $f^{\ell_{1}}\left(y^{\prime}\right)$. Choose $\gamma_{3}$ with $f^{\ell_{1}}\left(y^{\prime}\right)=\gamma_{1} \gamma_{3}$. Since $f^{k_{1}}\left(y^{\prime}\right)$ starts with a permutation of $\gamma \gamma_{3}$ is a prefix of this one, i.e. it is a also a suffix of $f^{\ell_{1}}(y)$ since this end in a permutation of $\gamma$. Transferred to the factor

285 of length $\left|\gamma_{1}\right|+\left|\gamma_{3}\right|$ from the point $|\gamma|$ on, the pattern $f^{a}\left(\gamma_{1}\right) f^{b}\left(\gamma_{3}\right)=f^{c}\left(\gamma_{3}\right) f^{d}\left(\gamma_{1}\right)$ for appropriate exponents $a, b, c, d \in \mathbb{N}_{0}$. Consequently $\gamma_{1}, \gamma_{3}$ are $[f]$-repetitions and since $y, y^{\prime}$ have only these as factors, $y, y^{\prime}$ are also $[f]$-repetitions. This property propagates via $x, x^{\prime}, x^{\prime \prime}$ to $u, v, w$.

Assume now $|x|<\left|x^{\prime}\right|$. Since permutations $x$ occur as a prefix and a suffix of permutations of $v$ there exists suffix $\hat{y}^{\prime}$ of $y^{\prime}$ and a prefix $\hat{y}$ of $y$ such that on the one hand $x$ equals $\hat{y}^{\prime}$ followed by a $y-y^{\prime}$ alternation and on the other hand a $y^{\prime}-y$-alternation followed by $\hat{y}^{\prime}$. Notice $|\hat{y}|=\left|\hat{y}^{\prime}\right|$. At the point $\alpha_{1}+|v|+\left|y^{\prime}\right|$ starts $f^{a \frac{r}{2}+1}(u)$ and since $x$ ends in a permutation of $\hat{y}^{\prime}$ there also starts $\hat{y}^{\prime}$ followed by another permutation of $y y^{\prime}$ (as a prefix of $\left.x^{\prime}\right)$. This implies $f^{a}\left(\hat{y}^{\prime}\right) f^{b}(y)=f^{c}(y) f^{d}\left(\hat{y}^{\prime}\right)$ for appropriate $a, b, c, d \in \mathbb{N}$. Hence $\hat{y}^{\prime}$ and $y$ are $\left[f, \gamma_{1}\right]$-repetition for a $\gamma \in \Sigma^{+}$. Analogously from the factor starting at $\left|\alpha_{1}\right|+|v|+|u|$ can be deduced that $y^{\prime}$ and $\hat{y}$ are $\left[f, \gamma_{2}\right]$-repetitions. Since $\hat{y}^{\prime}$ and $\hat{y}$ are suffixes and prefixes of $y^{\prime}, y$ resp. of the same length $\gamma_{1}$ and $\gamma_{2}$ are permutations of each other. With previous arguments $u, v, w$ are $\left[f, \gamma_{1}\right]$-repetitions (so $v=\gamma_{1}$ ).

case 2: Consider now $(r-1)|u|<|w|<r|u|$ and first assume $r$ to be odd. Choose $300 x^{\prime}, x \in \Sigma^{+}$with $f^{a_{r}}(u)=x^{\prime} x, x \in \operatorname{Pref}\left(f^{b_{2}}(w)\right)$, Since $r$ is odd, by the same arguments as before the point $\left|\alpha_{1}\right|+|v|$ is in the middle of $f^{a \frac{r}{2}+1}(u)$. Choose $y, y^{\prime} \in \Sigma^{+}$with $f^{a \frac{r}{2}+1}(u)=y y^{\prime}, y \in \operatorname{Suff}\left(f^{c_{1}}(v)\right)$, and $y^{\prime} \in \operatorname{Pref}\left(f^{c_{2}}(v)\right)$. By considering where the occurrences of permutations of $u$ occur in the permutations of $v$, there exist factors $\delta$ and $\delta^{\prime}$ of length less than $|y|=\left|y^{\prime}\right|$ such that $f^{c_{1}}(v)$ has a permutation of $y^{\prime}$ as a prefix, followed by $\delta$ followed by alternations of permutations of $y$ and $y^{\prime}$ (ending with the last $y$ ), and similarly, $f^{c_{2}}(v)$ begins with alternations of permutations of $y^{\prime}$ and $y$, followed eventually by $\delta^{\prime}$ and ending with a permutation of $y$ as a suffix. It is straightforward that $|\delta|=\left|\delta^{\prime}\right|$, and moreover, that $x f^{c_{1}-c_{2}}\left(y^{\prime}\right) \delta$ is the prefix of $f^{b_{2}}(w)$ of length $|u|$ and thus equal to a permutation of $u$. Furthermore, since $\delta^{\prime}$ is a prefix of a permutation of 310 $y$, and thus the factor of $v$ occurring after the prefix length $\left|y^{\prime}\right|$ is a permutation of both $\delta$ and $\delta^{\prime}$, these two factors must be permutations of each other.

Moreover, since permutations of $y$ and $\delta y$ both occur as prefixes of permutations of $u$ (observe e.g., the central and last occurrences of $u$ in $f^{b_{2}}(w)$ ), $y$ is of the form $f^{\ell_{1}}(\delta) \ldots f^{\ell_{k}}(\delta) \hat{\delta}$ for appropriate exponents $\ell_{i} \in \mathbb{N}$ with $i \in[k]$ and a prefix $\hat{\delta}$ of a 315 permutation of $\delta$. A symmetrical argument can be made to deduce that $y^{\prime}$ is of the form $\hat{\delta}^{\prime} f^{\ell^{\prime}}(\delta) \ldots f^{\ell^{\prime}}{ }^{\prime}(\delta)$ where $\hat{\delta}^{\prime}$ is a suffix of a permutation of $\delta$ and $\ell_{i}^{\prime} \in \mathbb{N}$ for $i \in[k]$. Hence $u$ is comprised of a series of permutations of $\delta$, followed by permutations of $\hat{\delta}$ 
and $\hat{\delta}^{\prime}$, followed by a further series of permutations of $\delta$. Recalling that $x f^{c_{1}-c_{2}}\left(y^{\prime}\right) \delta$ is a permutation of $u$, and additionally that $x$ is a suffix of a permutation of $u$, it follows by the (extended) Theorem of Fine and Wilf that $\hat{\delta}$ and $\hat{\delta}^{\prime}$ are empty. The fact that $u, v$ and $w$ are $[f, \delta]$-repetitions follows directly (so $v=\delta$ ).

case 3: Finally, consider the case that $(r-1)|u|<|w|<r|u|$ and $r$ is even. Note that w.l.o.g. we may assume that $u$ is $[f]$-primitive. Let $x, x^{\prime}$ be defined as before. Recall also that by the same reasoning as before, the point $2|w|-|v|$ is in the middle 325 of $f^{b_{2}-b_{1}}\left(\alpha_{1}\right)$ - in otherwords, the border between $f^{c_{1}}(v)$ and $f^{c_{2}}(v)$ is also a border between permutations of $u$. Since $w$ is covered by at least 4 permutations of $u$ (the last not being fully contained), we get that $f^{c_{2}}(v)$ is an alternating sequence of permutations of $x^{\prime}$ and $x$, ending finally with $x^{\prime}$, and thus has $f^{a}(x) f^{b}\left(x^{\prime}\right)$ as a suffix for some $a, b \in$ $\mathbb{N}_{0}$. On the other hand, $f^{c_{1}}(v)$ has a permutation of $u$, i.e. $f^{c}\left(x^{\prime}\right) f^{d}(x)$ for some $c, d \in$ $\mathbb{N}_{0}$ and hence we can apply Lemma 1 to obtain that $u$ is not $[f]$-primitive, which is a contradiction.

Obviously, if $(r-2)|w| \leq|u| \leq(r-1)|w|$, we can apply Lemma 9 to get the conclusion.

Lemma 12. If $u, v, w$ are words that satisfy Equation 7 for $t=2$ and $r, s \geq 3$, then there exists a word $v$ such that $u, v, w \in\left\{v, f(v), \ldots, f^{\text {ord }(f)-1}(v)\right\}^{*}$.

Proof. Firstly, it can be assumed that $u, v, w$ are [f]-primitive. If $u$ or $v$ are not $[f]$ primitive, then by replacing $u$ or, respectively, $v$ by their root in the new equation, a new equation of the same type $(t=2, r, s \geq 3)$ will be obtained. If $w$ is not $[f]$-primitive, then $w$ can be replaced by its root and an equation covered by Proposition 2 is obtained.

340 Without loss of generality, we can assume $\left|f^{a_{1}}(u) \ldots f^{a_{r}}(u)\right|>\left|f^{c_{1}}(v) \ldots f^{c_{s}}(v)\right|$. Also, by Lemma 9 it follows that if $\left|f^{a_{1}}(u) \ldots f^{a_{r}}(u)\right| \geq|u|+|w|$, then the statement follows, so for the rest of the proof it is assumed this does not hold. For the simplicity of the exposition, for $i \in[r]$, let $u_{i}=f^{a_{i}}(u)$ and, for $j \in[s]$, let $v_{j}=f^{c_{j}}(v)$.

The rest of the proof relies on a case analysis of the parity of the numbers $r$ and $s$.

case 1: $r$ and $s$ are even

It can be assumed that $r, s \geq 4$. Moreover we have $|u| \neq|v|$. Let $r=2 k$ and $s=2 p$. The border between $u_{k}$ and $u_{k+1}$ (which, occurs in the first $w$ ) is aligned to the border between $v_{p}$ and $v_{p+1}$, occurring in the second $w$. If $|u|>|v|$, then $f^{b_{1}-b_{2}}\left(v_{p}\right)$ is a suffix of $u_{k}$. This means also that there exists $\ell$ such that $f^{\ell}(v)$ is a suffix of $u_{k+1}$. As $u_{k+1}$ 350 is still fully contained in the first $w$, then the suffix $f^{\ell}(v)$ of $u_{k+1}$ is fully contained in a factor $v_{j} v_{j+1}$ of the second $w$. As $v$ is $[f]$-primitive, then the respective occurrence of $f^{\ell}(v)$ must be either aligned to $v_{j}$ or $v_{j+1}$. But this means that the end of $v_{p}$ is aligned to the end of $u_{k}$, and the end of $v_{j}$ or $v_{j+1}$ is aligned to the end of $u_{k+1}$. Consequently, $|v|$ divides $|u|$, so Lemma 6 can be applied to get the conclusion. The case $|v|>|u|$ 355 follows similarly, by noting that $u_{k}$ is a suffix of $v_{p}$, so $v_{p+1}$ also ends with $f^{\ell}(u)$. Using the $[f]$-primitivity of $u$, the conclusion follows.

case 2: $r$ is even and $s$ is odd

It can be assumed $r \geq 4$, as before. Let $r=2 k$ and $s=2 p+1$. The border between $u_{k}$ and $u_{k+1}$ (which occurs in the first $w$ ) is aligned to the middle point of $v_{p+1}$, occurring 

also inside $v_{p+2}$. Because $u$ is $[f]$-primitive, this occurrence of $f^{\ell}(u)$ must be aligned to an $u_{i}$. In that case, $|u|$ divides $|v|$, and the conclusion follows. If $|u|>|v|$ and $r \geq 5$,

in the second $w$. Let $v=x_{1} x_{2}$, with $\left|x_{1}\right|=\left|x_{2}\right|$. If $|u|=\left|x_{1}\right|$, the conclusion follows, as $|u|$ divides $|v|$. If $|u|>\left|x_{1}\right|$ (the case $|u|<\left|x_{1}\right|$ is symmetric), then $u_{k}$ ends with $f^{\ell_{1}}\left(x_{1}\right)$ and $u_{k+1}$ starts with $f^{\ell_{2}}\left(x_{2}\right)$. Thus, $u_{1}$ ends with $f^{\ell}\left(x_{1}\right)$ and $u_{2}$ starts with $f^{\ell^{\prime}}\left(x_{2}\right)$. Let $g=\left|f^{a_{1}}(u) \ldots f^{a_{r}}(u)\right|-|w|$ and $h=|u|-g$. If $h \geq\left|x_{1}\right|$, then $f^{\ell}\left(x_{1}\right) f^{\ell^{\prime}}\left(x_{2}\right)$ would occur either aligned to one of the $v_{i}$ 's or it would occur strictly inside a factor $v_{j} v_{j+1}$. The latter case leads to a contradiction with the $[f]$-primitivity of $v$, by Lemma 5. In the first case, it follows that $|v|$ divides $|u|$, so the conclusion would follow. If $h<\left|x_{1}\right|$ and $g+|v| \geq 2|u|$, it follows that $u_{2}$ is completely contained in $v_{1}$. So $f^{t}\left(u_{2}\right)$ occurs inside $v_{3}$. This latter occurrence of $f^{t}\left(u_{2}\right)$ must be aligned to an $u_{i}$ of the first $w$, or $u$ would not be $[f]$-primitive. But, in that case, $|u|$ divides $|v|$ and the conclusion follows. The remaining case $h<\left|x_{1}\right|$ and $g+|v|<2|u|$ is analysed further. Clearly, as $h<\left|x_{1}\right|$, we get $\left|x_{1}\right| \geq g+|v|-|u|-\left|x_{1}\right|>0$. Let $e=g+|v|-|u|-\left|x_{1}\right|$, and take $y_{1}$ to be the prefix of length $e$ of $v$. Consequently, the prefix $f^{\ell_{2}}\left(x_{2}\right)$ of $u_{k+1}$ would be followed, inside $u_{k+1}$, by $f^{\ell_{3}}\left(y_{1}\right)$. So, looking around the border between $f^{a_{1}}(u) \ldots f^{a_{r}}(u)$ and $f^{c_{1}}(v) \ldots f^{c_{s}}(v)$, it holds that an occurrence of $f^{\ell}\left(x_{1}\right) f^{\ell^{\prime}}\left(x_{2}\right) f^{\ell^{\prime \prime}}\left(y_{1}\right)$, from the first $w$, is aligned to an occurrence of $f^{\ell}\left(y_{1}\right) f^{c_{1}}(v)$. By Lemma 2 we get a contradiction to the primitivity of $v$. This concludes our analysis in this case.

case 3: $r$ is odd and $s$ is even

It can be assumed $s \geq 4$, as before. Let $r=2 k+1$ and $s=2 p$. The border between

3во $v_{p}$ and $v_{p+1}$ (which, occurs in the second $w$ ) is aligned to the middle point of $u_{k+1}$, occurring in the first $w$. Let $u=x_{1} x_{2}$, with $\left|x_{1}\right|=\left|x_{2}\right|$. If $|v|=\left|x_{1}\right|$, the conclusion follows, as $|v|$ divides $|u|$. If $|v|>\left|x_{1}\right|$, then $v_{p}$ ends with $f^{\ell_{1}}\left(x_{1}\right)$ and $v_{p+1}$ starts with $f^{\ell_{2}}\left(x_{2}\right)$. Thus, $v_{p+1}$ ends with $f^{\ell}\left(x_{1}\right)$ and $v_{p+2}$ starts with $f^{\ell^{\prime}}\left(x_{2}\right)$. This occurrence of $f^{\ell}\left(x_{1}\right) f^{\ell^{\prime}}\left(x_{2}\right)$ must be aligned to an occurrence of $u$ from the first $w$, or $u$ would not be primitive by Lemma 5. But, in that case, $|u|$ must divide $|v|$, and the conclusion follows. In the following, the case $|v|<\left|x_{1}\right|$ is considered. Once more,

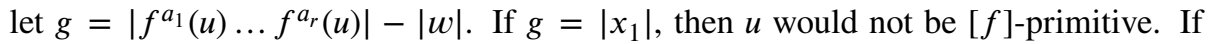
$g<\left|x_{1}\right|$, then $u_{r}$ contains an occurrence of $f^{\ell}(v)$ ending on its middle point (so inside the first $w$ ), which is the image of the occurrence of $v_{p}$ ending on the middle point of $u_{k+1}$. This $f^{\ell}(v)$ must be aligned to one of the $v_{j}$ 's of the second $w$, or $v$ would not be $[f]$-primitive. But in that case, $|v|$ divides $|u|$, and the conclusion follows. Finally, the case $g<\left|x_{1}\right|$ is considered. This means that on the middle point of $u_{r}$ an occurrence of $f^{\ell}(v)$ starts, inside the second $w$, which is the image of the occurrence of $v_{p+1}$ starting on the middle point of $u_{k+1}$. This is aligned to a factor of $u_{1}$. However, $u$, so also $395 u_{1}$, can be written as $f^{i_{0}}\left(v^{\prime \prime}\right) f^{i_{1}}(v) \ldots f^{i_{2 d}}(v) f^{i_{2 d+1}}\left(v^{\prime}\right)$ where $v^{\prime \prime}$ is a suffix of $v$ and $v^{\prime}$ is a prefix of $v$. Therefore, $f^{\ell}(v)$ must be aligned to one of the factors $f^{i_{j}}(v)$ of $u_{1}$. Again, this means that $|u|$ is divisible with $|v|$, and the conclusion would follow. This concludes the analysis of the case when $r$ is odd and $s$ even.

case 4: $r$ and $s$ are odd Let $r=2 k+1$ and $s=2 p+1$. The middle points of $u_{k+1}$ (which occurs in the first $w$ ) and $v_{p+1}$ (which occurs in the second $w$ ) are aligned. If $|v|>|u|$, then $f^{\ell}(u)$ occurs centred 
a similar argument can be applied, with the roles of $u$ and $v$ changed. So, the case $r=3$ remains to be considered. Let $u_{3}^{\prime}$ be the prefix of $u_{3}$ which is a suffix of the first $w$, and $u_{3}^{\prime \prime}$ its suffix that is a prefix of the second $w$. Also, $u_{1}=f^{i_{0}}\left(v^{\prime \prime}\right) f^{i_{1}}(v) \ldots f^{i_{2 d-1}}(v) f^{i_{2 d}}\left(v^{\prime}\right)$. Firstly, if $u_{2}$ is long enough so that it contains also the factors of the first $w$ aligned to $v_{p}$ and $v_{p+2}$ (that is, $d \geq 2$ ), then one of $u_{3}^{\prime}$ and $u_{3}^{\prime \prime}$ contains an occurrence of a factor $f^{\ell}(v)$, as well. If $u_{3}^{\prime}$ contains such a factor, because $v$ is [ $\left.f\right]$-primitive, it must be 410 aligned to one of the $v_{j}$ 's, so, just like before, $|v|$ divides $|u|$. The conclusion follows, in that case. If $u_{3}^{\prime \prime}$ contains a factor $f^{\ell}(v)$, then it must align to one of the factors $f^{i}(v)$ of $u_{1}$. This implies that $|v|$ divides $|u|$, and the conclusion follows as before. So, it remains to consider the case when $d=1$. If $\left|u_{3}^{\prime \prime}\right|+|v| \leq\left|u_{1}\right|$, then $v_{1}$ must be aligned to $f^{i_{1}}(v)$, so $|u|$ is divisible with $|v|$. The conclusion follows. We lastly consider the 415 case $\left|u_{3}^{\prime \prime}\right|+|v|>|u|$. So the end of $v_{1}$ corresponds to a position in $u_{2}$. As the factor aligned to $v_{p}$ is not contained in $u_{2}$, then $p=1$. It follows that $s=3$. In this case, let $u_{2}=y f^{b_{1}-b_{2}}\left(v_{2}\right) x$, where $|x|=|y|$. This means that $x f^{\ell_{0}}(y)$ is a prefix of $f^{b_{1}-b_{2}}\left(v_{3}\right)$. So, $f^{i_{1}}(v)$ has a prefix $f^{\ell_{1}}(x) f^{\ell_{2}}(y)$. Let $v_{\text {pref }}$ and $v_{\text {suff }}$ be the prefix and resp. suffix of $v$ of length $|x y|$. Since a permutation of $v$ is a suffix of $f^{i_{1}}(v) f^{a_{1}-a_{2}}(x) y$ and $|v| \geq|x y|$, ${ }_{420} f^{i_{1}}(v) f^{a_{1}-a_{2}}(x) y$ is a prefix of of $\left[f, v_{\text {pref }}\right]$-repetition and at the same time a suffix of a $\left[f, v_{\text {suff }}\right]$-repetition. Since $\left|f^{i_{1}}(v) f^{a_{1}-a_{2}}(x) y\right| \geq 2|x y|$ and $v_{\text {suff }}$ is a concatenation of permutations of $x, y$, by Lemma 5 it follows that $f^{a_{1}-a_{2}}(x) y$ and $v_{2}$ are $[f]$-repetitions of the same root. The only possibility for this to not lead to a contradiction is when the root is exactly $v$. This means $|y|<\left|v_{1}\right|<\left|f^{a_{1}-a_{2}}(x) y\right|$, so $v_{1}$ starts inside $f^{a_{1}-a_{2}}(x)$.

425 Therefore, $\left|u_{3}^{\prime \prime}\right|>|v|+|y|$, which leads to the fact that $u_{3}^{\prime \prime}$ contains $f^{a_{3}-a_{2}}\left(v_{2}\right)$ just before its suffix of length $|y|$. This factor must align to the $f^{a_{1}-a_{2}}\left(v_{2}\right)$ factor centred in $u_{1}$. Again, it follows that $|u|$ is divisible with $|v|$. So the conclusion follows once more. This concludes the analysis of this case, and the entire proof.

Lemma 13. If $u, v, w$ are words satisfying Equation 1, with $t=3$ and $r=s=2$, then 430 there exists a word $v$ such that $u, v, w \in\left\{v, f(v), \ldots, f^{\operatorname{ord}(f)-1}(v)\right\}^{*}$.

PROOF. In the setting of this lemma, Equation 1 becomes

$$
f^{a_{1}}(u) f^{a_{2}}(u) f^{c_{1}}(v) f^{c_{2}}(v)=f^{b_{1}}(w) f^{b_{2}}(w) f^{b_{3}}(w) .
$$

case 1: $|u|=|v|$. Then $|u|=|v|=\frac{3}{4}|w|$. Choose $w_{1}, w_{4} \in \Sigma^{\frac{1}{4}|w|}$ with

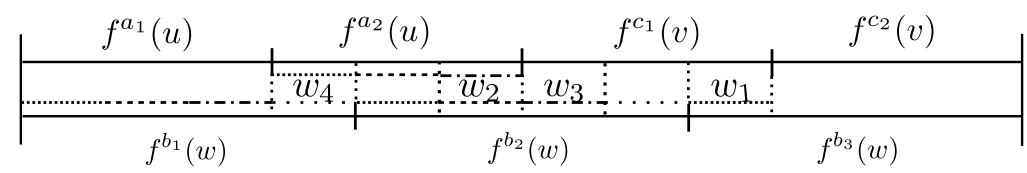

Figure 7: In the case of $r=s=2, t=3$ and $|u| \leq|v|$, the factors generated by the overlaps are permutations of each other.

$$
w_{1} \in \operatorname{Pref}\left(f^{b_{3}}(w)\right) \cap \operatorname{Suff}\left(f^{c_{1}}(v)\right) \text { and } w_{4} \in \operatorname{Suff}\left(f^{b_{1}}(w)\right) \cap \operatorname{Pref}\left(f^{a_{2}}(u)\right) .
$$


Since the points $2|u|$ and $\frac{3}{2}|w|$ are identical there exists $w_{2}, w_{3} \in \sum^{\frac{1}{4}|w|}$ such that

$$
f^{b_{2}}(w)=f^{b_{2}-b_{3}}\left(w_{1}\right) w_{2} w_{3} f^{b_{2}-b_{1}}\left(w_{4}\right)
$$

Transferring this to $f^{b_{1}}(w)$ implies $f^{a_{1}}(u)=f^{b_{1}-b_{3}}\left(w_{1}\right) f^{b_{1}-b_{2}}\left(w_{2} w_{3}\right)$. Hence

$$
\begin{aligned}
& f^{a_{2}-a_{1}+b_{1}-b_{3}}\left(w_{1}\right)=w_{4}, \\
& f^{a_{2}-a_{1}+b_{1}-b_{2}}\left(w_{2}\right)=f^{b_{2}-b_{3}}\left(w_{1}\right), \\
& f^{a_{2}-a_{1}+b_{1}-b_{2}}\left(w_{3}\right)=w_{2}
\end{aligned}
$$

\begin{tabular}{|c|c|c|c|}
\hline$f^{a_{1}}(u)$ & $f^{a_{2}}(u)$ & $f^{c_{1}}(v)$ & $f^{c_{2}}(v)$ \\
\hline רים & $\because: w_{1}$ & $w_{2}$ & \\
\hline
\end{tabular}

follow by $f^{a_{2}}(u)=f^{a_{2}-a_{1}+b_{1}-b_{3}}\left(w_{1}\right) f^{a_{2}-a_{1}+b_{1}-b_{2}}\left(w_{2} w_{3}\right)$. Thus, $w_{1}, w_{2}$, and $w_{3}$ are $\left[f, w_{4}\right]$-repetitions (so we can take $v=w_{4}$ ).

case 2: $|u|>|v|$ (w.l.o.g.). In the case $2|u|>2|w|$, we have $\left|\alpha_{1}\right|=2|u|>|u|+|w|>$

Figure 8: In the case of $r=s=2, t=3$ and $|v| \leq|u|$, the factors $x$ and $y$ form a similar pattern to the classical concept of borders of words.

$|u|+|w|-d_{u, w}$ and Lemma 8 is applicable. Consider $|w|<2|u|<2|w|$. Choose $w_{1}, w_{2}$ with $f^{b_{2}}(w)=w_{1} w_{2}$ and $w_{1}$ is a suffix of $f^{a_{2}}(u)$ and $w_{2}$ is a prefix of $f^{c_{1}}(v)$. Then

$$
|w|+\left|w_{1}\right|=2|u|>2|v|=|w|+\left|w_{2}\right|,
$$

which implies $\left|w_{1}\right|>\left|w_{2}\right|$. Choose $x, y \in \Sigma^{+}$with

$$
f^{a_{2}}(u)=y w_{1} \quad \text { and } \quad f^{c_{1}}(v)=w_{2} x .
$$

Thus, $x$ is a prefix of $f^{b_{3}}(w)$ and $y$ is a suffix of $f^{b_{1}}(w)$. Because we have $f^{a_{1}}(u)=$ $f^{a_{1}-a_{2}}(y) f^{a_{1}-a_{2}}\left(w_{1}\right)$ and $f^{c_{2}}(v)=f^{c_{2}-c_{1}}\left(w_{2}\right) f^{c_{2}-c_{1}}(x)$, it follows that

$$
f^{b_{3}}(w)=x f^{c_{2}-c_{1}}\left(w_{2}\right) f^{c_{2}-c_{1}}(x) \text { and } f^{b_{1}}(w)=f^{a_{1}-a_{2}}(y) f^{a_{1}-a_{2}}\left(w_{1}\right) y .
$$

As $|w|=\left|w_{1}\right|+\left|w_{2}\right|$, we get that $\left|w_{1}\right|=2|x|$ and $\left|w_{2}\right|=2|y|$, so $|x|>|y|$. Moreover Lemma 3 is applicable and there exist a suffix $x^{\prime}$ of a permutation of $x$, a prefix $y^{\prime}$ of a permutation of $y$, and $k_{1}, \ldots, k_{\ell}, k_{1}^{\prime}, \ldots, k_{\ell^{\prime}}^{\prime}, \ell, \ell^{\prime} \in \mathbb{N}$ such that

$$
w_{1}=f^{k_{1}}(y) \ldots f^{k_{\ell}}(y) y^{\prime} \text { and } w_{2}=x^{\prime} f^{k_{1}^{\prime}}(x) \ldots f^{k_{\ell^{\prime}}^{\prime}}(x) .
$$

Consider first that $|y|$ divides $|x|$. From $\left|w_{1}\right|=2|x|=\ell|y|+\left|y^{\prime}\right|$ if follows that $\left|y^{\prime}\right|=0$ and from $\left|w_{2}\right|=2|y|=\ell^{\prime}|x|+\left|x^{\prime}\right|$ it follows that $\left|x^{\prime}\right|=0$. Consequently $w_{1}$ and $w_{2}$ are $[f, y]$-repetitions and, because $f^{a_{2}}(u)=y w_{1} \quad$ and $f^{c_{1}}(v)=w_{2} x$, we obtain that $u, v, w$ are $[f, y]$-repetitions as well (so we can take $v=y$ ). 


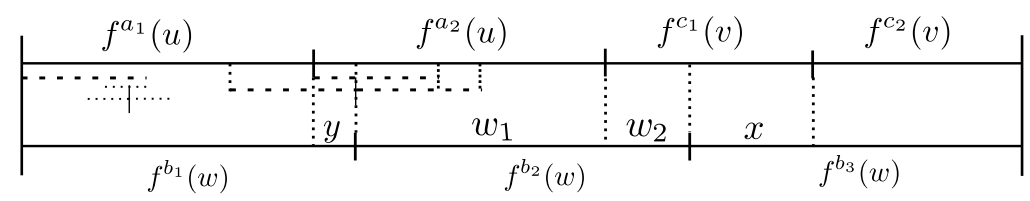

Figure 9: In the case of $r=s=2, t=3$ and $|v| \leq|u|$, the border-pattern shown in Fig. 8 1 leads to a 1-in-2 pattern.

Consider now the case when $|y|$ does not divide $|x|$. Then from the equality (2) we obtain $f^{b_{1}-b_{3}+c_{2}-c_{1}}(x) \in \operatorname{Suff}\left(f^{b_{1}}(w)\right)$ and $f^{b_{2}-b_{3}}(x) \in \operatorname{Pref}\left(f^{b_{2}}(w)\right)$. Moreover since $f^{b_{1}}(w)$ starts with $f^{b_{1}-b_{3}}(x), f^{a_{1}}(u)$ also does. This implies that $f^{a_{2}}(u)$ starts with $f^{a_{2}-a_{1}+b_{1}-b_{3}}(x)$. By $|u|>|v|$, it follows that $|w|-|x|=|v|<|u|$, i.e. the point $|w|-|x|$ is within $f^{a_{1}}(u)$ and the point $|w|$ is in $f^{a_{2}}(u)$ since $|w|>|u|$. Consequently there exists $u^{\prime}, u^{\prime \prime} \in \Sigma^{+}$with

$$
f^{b_{1}-b_{3}+c_{2}-c_{1}}(x) f^{b_{2}-b_{3}}(x)=u^{\prime} f^{a_{2}-a_{1}+b_{1}-b_{3}}(x) u^{\prime \prime} .
$$

435 Since $u^{\prime}$ and $u^{\prime \prime}$ are both not empty, $x$ can not be $[f]$-primitive by Lemma 4 and therefore there exists an $[f]$-primitive word $\delta \in \Sigma^{+},|\delta|<|x|$, and the integers $p_{1}, \ldots, p_{m}, m \in \mathbb{N}$ such that $x=f^{p_{1}}(\delta) \ldots f^{p_{m}}(\delta)$.

For getting an analogous result for $y$ consider the beginning of the words. Since $|y|$ is not a divisor of $|x|$, there exists an integer $v \in[\ell]$ such that the point $|x|$ is in $f^{a_{1}-a_{2}+k_{v}}(y)$. This sub-word is preceded by either $f^{a_{1}+k_{v-1}}(y)$ or $f^{a_{1}-a_{2}}(y)$. Moreover $f^{b_{1}}(w)$ ends in $f^{b_{1}-b_{3}+c_{2}-c_{1}}(x)$, i.e. the word ending in the point $|x|$ is $f^{b_{1}-b_{3}}(y)$. Consequently there exist $u^{\prime}, u^{\prime \prime} \in \Sigma^{+}$such that $u^{\prime} f^{b_{1}-b_{3}}(y) u^{\prime \prime}$ is

$$
f^{a_{1}+k_{v}}(y) f^{a_{1}+k_{v-1}}(y) \text { or } f^{a_{1}+k_{v}}(y) f^{a_{1}-a_{2}}(y) .
$$

Since $u^{\prime}$ and $u^{\prime \prime}$ are not empty, $y$ is not $[f]$-primitive and there exist an $[f]$-primitive word $\hat{\delta} \in \Sigma^{*}$ and $q_{1}, \ldots, q_{m^{\prime}}, m^{\prime} \in \mathbb{N}$ such that $y=f^{q_{1}}(\hat{\delta}) \ldots f^{q_{m^{\prime}}}(\hat{\delta})$.

Since $x$ has as non-empty prefix and as non-empty suffix images of $y$ under iterations of the permutation $f$, Lemma 3 shows that there exist an $[f]$-primitive $\tau \in \Sigma^{+}$, a suffix $\tau^{\prime} \in \Sigma^{+}$of $\tau$, and $p_{1}, \ldots, p_{m}, p_{m+2}, q_{1}, \ldots, q_{m}, q_{m+1}, m \in \mathbb{N}$ with

$$
y=f^{q_{1}}(\tau) \ldots f^{q_{m}}(\tau) f^{q_{m+1}}\left(\tau^{\prime}\right) \text { and } x=f^{p_{1}}(\tau) \ldots f^{p_{m+1}}(\tau) f^{p_{m+2}}\left(\tau^{\prime}\right)
$$

440 Using this and Theorem 4 for $x=f^{p_{1}}(\delta) \ldots f^{p_{m}}(\delta)$, we get that $\tau^{\prime}=\epsilon$ and $\delta$ is the image of $\tau$ under an iteration of $f$. The same holds, clearly, for $\hat{\delta}$.

Thus $|\tau|$ divides both $|y|$ and $\left|w_{1}\right|=2|x|$. As $w_{1}=f^{k_{1}}(y) \ldots f^{k_{\ell}}(y) y^{\prime}$, we get that $y^{\prime}$ is empty and $w_{1}$ is an $[f, \tau]$-repetition. Same holds for $w_{2}$. So $w, u, v$ are $[f, \tau]$ repetitions and we can take $v=\tau$.

This concludes the proof of the lemma.

Lemma 14. If $u, v, w$ are words satisfying Equation 1 with $r=2$ and $s=t=3$, then there exists a word $v$ such that $u, v, w \in\left\{v, f(v), \ldots, f^{\operatorname{ord}(f)-1}(v)\right\}^{*}$. 
PROOF. If $3|v|<|w|$ then $\alpha_{1}$ and $\beta$ share a common prefix of length at least $|w|+|u|>$ $|w|+|u|-d_{u, w}$ by $2|u|>2|w|$. Thus by Lemma 9 the claim is proven.

We split the remaining analysis in two cases.

case 1: If $3|v| \geq \frac{3}{2}|w|$, then $|v| \geq \frac{|w|}{2}$, so $3|v| \geq|w|+|v|$. Thus, we can apply Lemma 9 because $\alpha_{2}$ and $\beta$ share a long enough suffix, and the conclusion follows.

case 2: $|w|<3|v|<\frac{3}{2}|w|$.

Choose $v_{1}, v_{2} \in \Sigma^{+}$with $f^{c_{1}}(v)=v_{1} v_{2}$ and $v_{1}$ is a suffix of $f^{b_{2}}(w)$ and $v_{2}$ is a

\begin{tabular}{|c|c|c|c|c|c|}
\hline$f^{a_{1}}(u)$ & $f^{a_{2}}(u)$ & & & $f^{c_{2}}(v)$ & $f^{c_{3}}(v)$ \\
\hline$-1---1$ & $u_{1} u_{2}$ & $v_{1}$ & $v_{2}$ & & $\ldots \ldots . .$. \\
\hline
\end{tabular}

Figure 10: In the case $r=2$ and $s=t=3$ such that the point $2|u|$ is beyond the point $\frac{3}{2}|w|$ the repetitionenforcing patterns depend on the factors $v_{1}, v_{2}$ which build the first $v$.

prefix of $f^{b_{3}}(v)$. Furthermore choose $u_{1}, u_{2} \in \Sigma^{+}$with $f^{a_{2}}(u)=u_{1} u_{2}$ and $u_{1}$ is a suffix of $f^{b_{1}}(w)$ and $u_{2}$ is a prefix of $f^{b_{2}}(w)$. Then $f^{c_{3}-c_{2}}\left(v_{2}\right), f^{b_{3}-b_{1}}\left(u_{1}\right)$ are suffixes of $f^{b_{3}}(w)$. Moreover since $f^{b_{3}}(w)$ starts with $v_{2}$ and $f^{a_{2}}(u)$ starts with $u_{1}$ the factors $f^{b_{2}-b_{3}}\left(v_{2}\right)$ and $f^{a_{1}-a_{2}+b_{2}-b_{1}}\left(u_{1}\right)$ are prefixes of $f^{b_{2}}(w)$. Since $f^{b_{2}}(w)$ starts with $u_{2}$ the factors $f^{b_{2}-b_{3}}\left(v_{2}\right)$ and $f^{a_{1}-a_{2}+b_{2}-b_{1}}\left(u_{1}\right)$ share a prefix with $u_{2}$ as well. By $3|v|<\frac{3}{2}|w|$, it follows that $2|u|>\frac{3}{2}|w|$, i.e. $|u|>\frac{3}{4}|w|$. From the definition of $u_{1}$ we obtain $\left|u_{1}\right|<\frac{1}{4}|w|$. This implies $\left|u_{2}\right|>\frac{1}{2}|w|$, because $|w|=2\left|u_{1}\right|+\left|u_{2}\right|$. This results in $\left|u_{1}\right|<\left|u_{2}\right|$. Again, by comparing different permutations of $|w|$, we get $\left|v_{2}\right|<\left|u_{2}\right|$, since $\left|u_{2}\right|+\left|v_{1}\right|=|w|=3\left|v_{2}\right|+2\left|v_{1}\right|$. Thus $f^{b_{2}-b_{3}}\left(v_{2}\right)$ and $f^{a_{1}-a_{2}+b_{2}-b_{1}}\left(u_{1}\right)$ are prefixes of $u_{2}$.

Also, as $f^{a_{2}}(u)$ is a prefix of $u_{1} f^{b_{2}}(w)$, which starts with $u_{1} f^{b_{2}+a_{1}-b_{1}}\left(u_{1}\right)$, we immediately get the existence of an $\ell \in \mathbb{N}_{0}$ and a prefix $u_{1}^{\prime}$ of the image of $u_{1}$ under an iteration of $f$ with $u \in\left\{f^{k}\left(u_{1}\right) \mid k \in[\operatorname{ord}(f)]_{0}\right\}^{\ell} u_{1}^{\prime}$.

case 2.a: $\left|v_{1}\right| \leq\left|v_{2}\right|$. Consider the longest prefix $y$ of $f^{b_{3}}(w)$ such that $|y|$ is di-

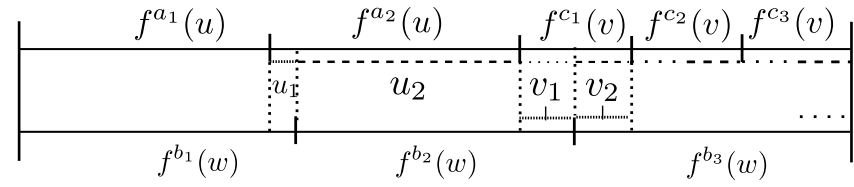

Figure 11: In the sub-case of Fig. 10 in which $\left|v_{1}\right|=\left|v_{2}\right|$, both are permutations of each other and the root is a suffix of the first $w$.

visible by $\left|u_{1}\right|$ and $|y|<|w|-|v|+\left|u_{1}\right|$. Since $\left|v_{1}\right|=2\left|u_{1}\right|$ and $|v|>2\left|u_{1}\right|$ we have $|w|-|v|+\left|u_{1}\right|<|w|-\left|u_{1}\right|=|u|$. This implies that $y$ is an $\left[f, u_{1}\right]$-repetition. If $|v|$ is divisible by $\left|u_{1}\right|$ it is easy to see that $\mathrm{v}$ and $\mathrm{w}$ are $\left[f, u_{1}\right]$-repetitions. Since $f^{b_{1}}(w)=f^{a_{1}}(u) u_{1}, u$ is also $\left[f, u_{1}\right]$-repetition. If $\mid \mathrm{vl}$ is not divisible by $\left|u_{1}\right|$ we have 


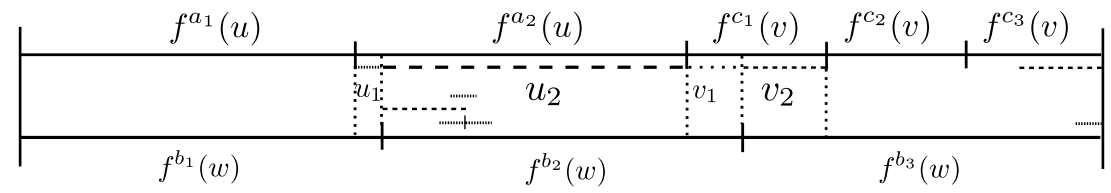

Figure 12: In the sub-case of Fig. 10 in which $\left|v_{1}\right|<\left|v_{2}\right|$, either the root of case a is again a root or this factor is a repetition of a smaller root.

$|y|>|w|-|v|$. Since a permutation of $u_{1}$ is a suffix of $f^{c_{2}}(v)$, we can see the 1 -in-2 pattern of $u_{1}$ at the end of $y$. Hence, there is a $\gamma$ such that $u_{1}$ and also $v$ are $[f, \gamma]$-repetitions. In a similar way to the above case, we can show that $u, v$, and $w$ are $[f, \gamma]$-repetitions. We can take thus $v=\gamma$.

case 2.b: $\left|v_{1}\right|<\left|v_{2}\right|$ Suppose $\left|v_{2}\right| \geq \ell\left|u_{1}\right|$. Then

$$
(\ell+1)\left|u_{1}\right|+\left|u_{1}^{\prime}\right|=2\left|v_{1}\right|+3\left|v_{2}\right| \geq 4\left|u_{1}\right|+3 \ell\left|u_{1}\right|=(3 \ell+4)\left|u_{1}\right|,
$$

i.e. $\left|u_{1}^{\prime}\right| \geq(2 \ell+3)\left|u_{1}\right|$. Since $\left|v_{2}\right|<\ell\left|u_{1}\right|$ there exist $k_{1}<\ell$ such that the point $|w|+\left|v_{2}\right|$ (the end of $f^{b_{2}-b_{3}}\left(v_{2}\right)$ ) is within $f^{k_{1}}\left(u_{1}\right)$. Since $f^{a_{1}-a_{2}}\left(u_{1}\right)$ is a suffix of $f^{b_{1}-b_{3}}\left(v_{2}\right), f^{a_{1}-a_{2}-b_{1}+b_{2}}\left(u_{1}\right)$ is a suffix of $f^{b_{1}-b_{3}}\left(v_{2}\right)$. Choose $x, y \in \Sigma^{*}, k_{2} \in \mathbb{N}$ with

$$
f^{k_{1}}\left(u_{1}\right) f^{k_{2}}\left(u_{1}\right)=x f^{a_{1}-a_{2}-b_{1}+b_{2}}\left(u_{1}\right) y .
$$

If $u_{1}$ is primitive, then $\left|u_{1}\right|$ divides $\left|v_{2}\right|$ and $v_{2}$ is an $\left[f, u_{1}\right]$-repetition. Since $f^{b_{2}}(w)$ ends in $f^{b_{2}-b_{3}+c_{3}}\left(v_{2}\right)$ and $\left|v_{1}\right|=2\left|u_{1}\right|$ holds, $v_{1}$ has the same property. Consequently $w$ is an $[f, w]$-repetition and by $f^{a_{1}}(w)=f^{a_{1}-a_{2}}\left(u_{1}\right) f^{a_{1}-a_{2}}\left(u_{2}\right) u_{1}, u_{2}$ is also one. If $u_{1}$ is not primititve, there exists a $\gamma \in \Sigma^{+}$such that $u_{1}$ is an $[f, \gamma]$-repetition. This implies the existence of a $k_{3} \in \mathbb{N}$ with $u \in\left\{f^{\ell}(\gamma) \mid \ell \in[\operatorname{ord}(f)]_{0}\right\}^{k_{3}} \gamma^{\prime}$ for a prefix $\gamma^{\prime}$ of a permutation of $\gamma$. Choose $v_{1}, \ldots, v_{k_{2}}, \mu_{1}, \ldots, \mu_{k_{1}}, \xi_{1}, \xi_{2} \in[\operatorname{ord}(f)]_{0}$ with

$$
\begin{gathered}
f^{\nu_{1}}(\gamma) \ldots f^{\nu_{k_{2}}}(\gamma) f^{\xi_{1}}\left(\gamma^{\prime}\right) f^{\mu_{1}}(\gamma) \ldots f^{\mu_{k_{1}}}(\gamma) \\
=w= \\
f^{\nu_{1}}(\gamma) \ldots f^{\nu_{k_{2}-k_{1}}}(\gamma) f^{\xi_{2}}\left(\gamma^{\prime}\right) x f^{\mu_{1}}(\gamma) \ldots f^{\mu_{k_{1}}}(\gamma) .
\end{gathered}
$$

465 Since $\gamma$ is $[f]$-primitive, Lemma 4 implies $\gamma^{\prime}=\varepsilon$. Consequently $u, v, w$ are $[f]$ repetitions.

Summing up the conclusions of case 1 and of cases 2.a and 2.b, the conclusion of the lemma follows.

Following Proposition 2 and the series of Lemmas $10|11| 12|13| 14 \mid j u s t$ shown, we conclude with the following main result.

Theorem 5. If $u, v, w$ are words satisfying Equation 1 for $t, r, s \geq 2$, then there exists $a$ word $v$ such that $u, v, w \in\left\{v, f(v), \ldots, f^{\operatorname{ord}(f)-1}(v)\right\}^{*}$. 


\section{The Antimorphic Case}

Firstly, note that the results of Lemma 4 do not hold in the case of antimorphic

Remark 2. Consider the equation $f^{a}(w) f^{b}(w)=x f^{c}(w) y$, where $f$ is an antimorphic permutation. The following counterexamples show that this equation is not repetition enforcing, no matter the values of $a, b, c$; if $\Sigma=\{\mathrm{a}, \mathrm{b}\}$ and $f$ being the mirror image on $\Sigma^{*}$. Let $w=$ aaba, which is not a $[f]$-repetition. However, for $a, c$ even and $b$ odd, we have $f^{a}(w) f^{b}(w)=$ aabaabaa $=\operatorname{aab} f^{c}(w)$ a. We will show, in Lemma 16 . by a duality argument that the equation $f^{a}(w) f^{b}(w)=x f^{c}(w) y$ when $a$ is odd and $b, c$ even, $a, c$ odd and $b$ even, and $a, c$ even and $b$ odd, does not necessarily have solutions which are $[f]$-repetitions. If $a, b$ are even and $c$ odd, for the same $w$ and $f$, we have $f^{a}(w) f^{b}(w)=$ aabaaaba $=\mathrm{a} f^{c}(w)$ aba. Again, it is an immediate consequence that when $a, b$ are odd and $c$ is even then $f^{a}(w) f^{b}(w)=x f^{c}(w) y$ does not necessarily have solutions which are $[f]$-repetitions.

Following the ideas of [19], an extension of Lemma 4 to the antimorphic case can be obtained by considering equations of the form

$$
f^{a_{1}}(w) f^{a_{2}}(w) f^{a_{3}}(w)=x f^{b_{1}}(w) f^{b_{2}}(w) y
$$

for $f$ antimorphic permutation on $\Sigma, a_{1}, a_{2}, a_{3}, b_{1}, b_{2} \in \mathbb{N}_{0}, w, x, y \in \Sigma^{*}, 0<|x|,|y|<$ $|w|$. Our goal is to identify under which restrictions on $a_{1}, a_{2}, a_{3}, b_{1}, b_{2}$ the equation above enforces that $x, y, w$ are $[f]$-repetitions.

The main difference to the morphic case is that when iterating the application of an antimorphic permutation $f$ on a word $w, f^{i}(w)$ preserves the order of letters of $w$ when $i$ is even, and reverses it when $i$ is odd; in the morphic case, the order was preserved for all exponents. Therefore, it seems a good approach to classify the equations considered above by the parity of their exponents.

In the following, $e$ (from even) and $o$ (from odd) are used for 0 and 1 resp., for convenience. Moreover for each number $a$ let $\bar{a}$ denote its residue class modulo 2.

Definition 1. Let $f$ be an antimorphic permutation over an alphabet $\Sigma$. Define the set

$$
\begin{aligned}
\mathcal{E}_{f}:= & \left\{f^{a_{1}}(w) f^{a_{2}}(w) f^{a_{3}}(w)=x f^{b_{1}}(w) f^{b_{2}}(w) y \mid\right. \\
& \left.w, x, y \in \Sigma^{*}, 0<|x|,|y|<|w|, a_{1}, a_{2}, a_{3}, b_{1}, b_{2} \in \mathbb{N}_{0}\right\} .
\end{aligned}
$$

The equations

$$
\begin{aligned}
E & : f^{a_{1}}(w) f^{a_{2}}(w) f^{a_{3}}(w)=x f^{b_{1}}(w) f^{b_{2}}(w) y \text { and } \\
E^{\prime}: f^{a_{1}^{\prime}}(w) f^{a_{2}^{\prime}}(w) f^{a_{3}^{\prime}}(w) & =x^{\prime} f^{b_{1}^{\prime}}(w) f^{b_{2}^{\prime}}(w) y^{\prime}
\end{aligned}
$$

are called equivalent (denoted $E \sim E^{\prime}$ ) if $\left(\overline{a_{1}}, \overline{a_{2}}, \overline{a_{3}}, \overline{b_{1}}, \overline{b_{2}}\right)=\left(\overline{a_{1}^{\prime}}, \overline{a_{2}^{\prime}}, \overline{a_{3}^{\prime}}, \overline{b_{1}^{\prime}}, \overline{b_{2}^{\prime}}\right)$. A class of equivalent equations will be denoted by the quintuple $\left(\overline{a_{1}}, \overline{a_{2}}, \overline{a_{3}} \mid \overline{b_{1}}, \overline{b_{2}}\right)$. Such a class (resp., quintuple) is called repetition enforcing if every equation in this class has only solutions which are $[f]$-repetitions. 
Remark 3. Note that $\sim$ is an equivalence relation. Consequently, the quotient set $\mathcal{E}_{f} / \sim$ is well defined. Since the elements of $\mathcal{E}_{f}$ are defined by five parameters, which are further reduced by the factorization w.r.t. $\sim$ to their canonical representative from $\mathbb{Z}_{2}$, $\mathcal{E}_{f} / \sim$ has only 32 elements. Since all equivalent equations are associated to the same quintuple of elements of $\mathbb{Z}_{2}$, these quintuples can be used as canonical representatives for the classes of $\mathcal{E}_{f} / \sim$.

In order to further group together classes of equation, it is worth noting the following.

Lemma 15. Let $f$ be an antimorphic permutation on $\Sigma$. Consider for fixed $k \in \mathbb{N}$ the equations

$$
\begin{aligned}
f^{i_{1}}(w) \cdots f^{i_{k}}(w) & =x f^{j_{1}}(w) \cdots f^{j_{k-1}}(w) y, 0<|x|,|y|<|w| \\
f^{i_{1}-1}(u) \cdots f^{i_{k}-1}(u) & =x f^{j_{1}-1}(u) \cdots f^{j_{k-1}-1}(u) y, 0<|x|,|y|<|u| \\
f^{i_{k}+1}(w) \cdots f^{i_{1}+1}(w) & =f(y) f^{j_{k-1}+1}(w) \cdots f^{j_{1}+1}(w) f(x), 0<|x|,|y|<|w| \\
f^{i_{k}}(u) \cdots f^{i_{1}}(u) & =f(y) f^{j_{k-1}}(u) \cdots f^{j_{1}}(u) f(x), 0<|x|,|y|<|u|
\end{aligned}
$$

All the solutions of equation ( $i$ ) are $[f]$-repetitions if and only if all solutions of equation (j) are $[f]$-repetitions, with $3 \leq i, j \leq 6$.

PROOF. Firstly, note that if $w$ is an [f]-repetition, then there exists $\gamma \in \Sigma^{*}$ and $p \geq 2$, such that $w=f^{a_{1}}(\gamma) \cdots f^{a_{p}}(\gamma)$. Consequently, $f(w)$ and $f^{-1}(w)$ are $[f]$-repetitions.

Further, we show that all solutions of equation (3) are $[f]$-repetitions if and only ${ }_{515}$ if all solutions of equation $(j)$ are $[f]$-repetitions, with $3 \leq j \leq 6$. Equation (4) is obtained from equation (3) by taking $u=f(w)$, and $(3)$ from (4) by taking $w=f^{-1}(u)$. Equation (5) is obtained from (3) by applying $f$, and (3) is obtained from the (5) by applying $f^{-1}$. Finally, equation (6) is obtained from equation 3 (and viceversa) by composing the previous two transformations.

Following the ideas of Lemma 15 it makes sense to define the following relation.

Definition 2. Two elements $E=\left(a_{1}, a_{2}, a_{3} \mid b_{1}, b_{2}\right)$ and $E^{\prime}=\left(a_{1}^{\prime}, a_{2}^{\prime}, a_{3}^{\prime} \mid b_{1}^{\prime}, b_{2}^{\prime}\right)$ of $\mathcal{E}_{f} / \sim$ are called dual $\left(E_{1} \circ E_{2}\right)$ if either $E=E^{\prime}$ or one of the following cases holds:

1. $\left(\overline{a_{1}^{\prime}}, \overline{a_{2}^{\prime}}, \overline{a_{3}^{\prime}} \mid \overline{b_{1}^{\prime}}, \overline{b_{2}^{\prime}}\right)=\left(\overline{a_{3}+1}, \overline{a_{2}+1}, \overline{a_{1}+1} \mid \overline{b_{2}+1}, \overline{b_{1}+1}\right)$ (equating to the application of $f$ to $E$ )

2. $\left(\overline{a_{1}^{\prime}}, \overline{a_{2}^{\prime}}, \overline{a_{3}^{\prime}} \mid \overline{b_{1}^{\prime}}, \overline{b_{2}^{\prime}}\right)=\left(\overline{a_{1}+1}, \overline{a_{2}+1}, \overline{a_{3}+1} \mid \overline{b_{1}+1}, \overline{b_{2}+1}\right)$ (equating to $f^{z}(w)=$ $f^{z-1}(f(w))$ for $\left.z \in \mathbb{Z}_{2}\right)$

3. $\left(\overline{a_{1}^{\prime}}, \overline{a_{2}^{\prime}}, \overline{a_{3}^{\prime}} \mid \overline{b_{1}^{\prime}}, \overline{b_{2}^{\prime}}\right)=\left(\overline{a_{3}}, \overline{a_{2}}, \overline{a_{1}} \mid \overline{b_{2}}, \overline{b_{1}}\right)$ (equating to the application of (1) and (2))

Remark 4. Since $\circ \circ$ is also an equivalence relation the above mentioned 32 classes 
can be reduced to the following 10 classes of $\left(\mathcal{E}_{f} / \sim\right) / \circ \circ$

$$
\begin{aligned}
& {[(e, e, e \mid e, e)]=\{(e, e, e \mid e, e),(o, o, o \mid o, o)\}} \\
& {[(e, e, e \mid e, o)]=\{(e, e, e \mid e, o),(o, o, o \mid e, o),(o, o, o \mid o, e),(e, e, e \mid o, e)\}} \\
& {[(e, e, e \mid o, o)]=\{(e, e, e \mid o, o),(o, o, o \mid e, e)\}} \\
& {[(e, e, o \mid e, e)]=\{(e, e, o \mid e, e),(e, o, o \mid o, o),(o, o, e \mid o, o),(o, e, e \mid e, e)\}} \\
& {[(e, e, o \mid e, o)]=\{(e, e, o \mid e, o),(e, o, o \mid e, o),(o, o, e \mid o, e),(o, e, e \mid o, e)\}} \\
& {[(e, e, o \mid o, e)]=\{(e, e, o \mid o, e),(e, o, o \mid o, e),(o, o, e \mid e, o),(o, e, e \mid e, o)\}} \\
& {[(e, e, o \mid o, o)]=\{(e, e, o \mid o, o),(e, o, o \mid e, e),(o, o, e \mid e, e),(o, e, e \mid o, o)\}} \\
& {[(e, o, e \mid e, e)]=\{(e, o, e \mid e, e),(o, e, o \mid o, o)\}} \\
& {[(e, o, e \mid e, o)]=\{(e, o, e \mid e, o),(o, e, o \mid e, o),(o, e, o \mid o, e),(e, o, e \mid o, e)\}} \\
& {[(o, e, o \mid e, e)]=\{(o, e, o \mid e, e),(e, o, e \mid o, o)\}}
\end{aligned}
$$

For convenience we will enumerate the classes and dedicate one representative for each class:

$$
\begin{array}{llll}
(c 1) & {[(e, e, e \mid e, e)]} & {[(e, e, e \mid e, o)]} & (c 2) \\
(c 3) & {[(e, e, e \mid o, o)]} & {[(e, e, o \mid e, e)]} & (c 4) \\
(c 5) & {[(e, e, o \mid e, o)]} & {[(e, e, o \mid o, e)]} & (c 6) \\
(c 7) & {[(e, e, o \mid o, o)]} & {[(e, o, e \mid e, e)]} & (c 8) \\
(c 9) & {[(e, o, e \mid e, o)]} & {[(o, e, o \mid e, e)]} & (c 10)
\end{array}
$$

The following lemma is a direct consequence of Lemma 15 $E_{1}$ is repetition-enforcing then $E_{2}$ is as well.

For eight of the ten classes of $(\mathcal{E} / \sim) / \circ \multimap$ it will be shown that they are repetitionenforcing. In the remaining cases, counter-examples will be given.

Lemma 17. Classes (c3) (represented by $(e, e, e \mid o, o))$ and (c7) (represented by $(e, e, o \mid$ $\left.{ }_{535} o, o\right)$ ) are not repetition-enforcing.

PROOF. Equations of these classes that have solutions which are not $[f]$-repetitions can be obtained by extending the examples in Remark 2

Consider $w=$ aaba and $f$ the mirror image on $\Sigma=\{\mathrm{a}, \mathrm{b}\}$. Although $w$ is not an $[f]$-repetition, we have the following: $w w w=$ aabaaabaaaba $=\mathrm{a} f(w) f(w)$ aba, so 40 (c3) is not repetition enforcing.

Also, the following is true $w w f(w)=$ aabaaabaabaa $=\mathrm{a} f(w) f(w)$ baa, so $(c 7)$ is not repetition enforcing.

For some classes the repetition enforcement can be proven by Lemma 4 from the morphic case. This is possible since the word $f^{b_{1}}(w)$ occurs inside $f^{a_{1}}(w) f^{a_{2}}(w)$ and ${ }_{545} a_{1}, a_{2}, b_{1}$ are even (for short, e occurs in ee) in all equations contained in the classes ( $c 1$ ), $(c 2),(c 4)$, and (c5). In class (c10) we again have that $e$ occurs in $e e: f^{a_{2}}(w)$ is a factor 
of $f^{b_{1}}(w) f^{b_{2}}(w)$, and $a_{2}, b_{1}, b_{2}$ are all even. Class (c8) may appear to be different but in fact it contains a similar structure. The characteristic of the aforementioned pattern is, that - neglecting the permutations for a moment - a word is split into $w=u v$ and $u$ occurs also as a suffix and $v$ also as a prefix. Having a deeper look into the representative of class (c8) reveals that a prefix $u$ of $f^{b_{1}}(w)$ is a suffix of $f^{a_{1}}(w)$ and a suffix $v$ of $f^{b_{2}}(w)$ is a prefix of $f^{a_{3}}(w)$ with $|u v|=|w|,|u|=|y|$, and $|v|=|x|$. Thus, $f^{b_{1}}(w)=$ $u f^{b_{1}-b_{2}}(v)$ is a factor of $f^{a_{1}}(w) f^{a_{3}}(w)$ and $a_{1}, a_{3}, b_{1}$ are all even. Accordingly, the following lemma holds.
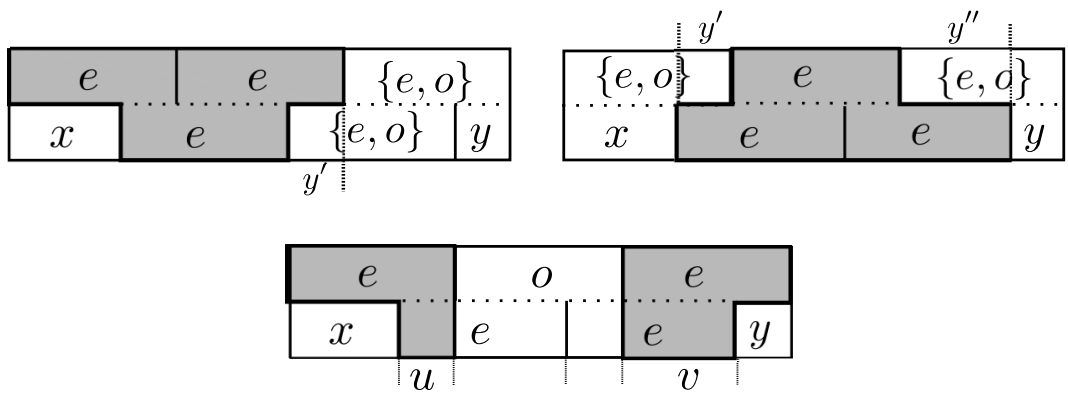

Figure 13: With the aforementioned abbreviations $e$ and $o$ for an arbitrary even resp. odd permutation of $f$ the classes $c 1, c 2, c 4$, and $c 5$ are given by the first picture. Class $c 10$ is represented by the second picture and the picture below shows that the necessary 1-in-2 pattern (here visualised as a grey "T") occurs if the middle part is ignored.

Lemma 18. The classes (c1), (c2), (c4), (c5), (c8) and (c10) are repetition-enforcing.

Before showing that class $(c 9)$ is also repetition enforcing, several more definitions are needed.

Definition 3. If $w=f^{i_{1}}(s) f^{i_{2}}(s) \cdots f^{i_{k}}(s)$, for some $s \in \Sigma^{*}$ and $k \geq 1$, and $i_{j} \not_{2} i_{j+1}$ for all $j \in[k-1]$, then $w$ is called an alternating $[f, s]$-repetition. If the word $w$ is an alternating $[f, s]$-repetition for some $s$, but this word $s$ is not important to us, then we just say that $w$ is an alternating $[f]$-repetition.

It can be shown that if $w$ is an alternating $[f, s]$-repetition then $f^{a_{1}}(w) f^{a_{2}}(w) f^{a_{3}}(w)$ is also an alternating $[f, s]$-repetition, provided that $a_{1} \equiv_{2} a_{3} \equiv_{2} a_{2}$. Indeed, assume $a_{1}, a_{3}$ are even, and $a_{2}$ is odd (the other case is similar). If $w=f^{i_{1}}(s) f^{i_{2}}(s) \cdots f^{i_{k}}(s)$, for some $s \in \Sigma^{*}$ and $k \geq 1$, and $i_{j} \not \equiv_{2} i_{j+1}$ for all $1 \leq j \leq k-1$, then

$$
\begin{aligned}
f^{a_{1}}(w) f^{a_{2}}(w) f^{a_{3}}(w)= & f^{a_{1}+i_{1}}(s) \cdots f^{a_{1}+i_{k}}(s) . \\
& f^{a_{2}+i_{k}}(s) \cdots f^{a_{2}+i_{1}}(s) . \\
& f^{a_{3}+i_{1}}(s) \cdots f^{a_{3}+i_{k}}(s) .
\end{aligned}
$$

As $a_{1}+i_{k}$ has a different parity than $a_{2}+i_{k}$, and $a_{2}+i_{1}$ has a different parity than $a_{3}+i_{1}$, the claim follows. 
Next, it is shown that class ( $c 9)$ is repetition enforcing, and, moreover, that if $w$ is a solution of an equation from the respective class, then $w$ is an alternating $[f, s]$ repetition for some word $s$.

Lemma 19. Class (c9) is repetition-enforcing. More precisely, if

$$
f^{a_{1}}(w) f^{a_{2}}(w) f^{a_{3}}(w)=x f^{b_{1}}(w) f^{b_{2}}(w) y
$$

with $x, y \neq \varepsilon$ and $\left(a_{1}, a_{2}, a_{3} \mid b_{1}, b_{2}\right)$ in class (c9), then there exists $s \in \Sigma^{*}$ such that $x f^{b_{1}}(w)$ and $x w$ are alternating $[f, s]$-repetitions.

ProOF. We will show this result by induction on the length of $w$. The base case is $|w|=$ 570 2. In this case $f^{a_{1}}(w)=x f^{b_{1}-a_{1}}(x)$, and $f^{a_{3}-a_{1}}(x)=f^{b_{2}-a_{1}}(x)$. Thus, $x=f^{b_{2}-a_{3}}(x)$, and $f^{a_{1}}(w)=x f^{b_{1}+b_{2}-a_{1}-a_{3}}(x)$. This shows that $w$ is an alternating $[f, x]$-repetition.

The induction hypothesis is that the statement holds for $|w| \leq n$ and in the following it is shown the statement also holds for $|w|=n+1$. As $f^{a_{1}}(w)$ is a prefix of $x f^{b_{1}}(w)$, and both $a_{1}$ and $b_{2}$ are even, we get by Lemma 3 that $w=f^{i_{1}}(x) \ldots f^{i_{k}}(x) x^{\prime}$, where $575 i_{1}, \ldots, i_{k}$ are alternating and $x^{\prime}$ is a prefix of $f^{i_{k+1}}(x)$ for some even $i_{k+1},\left|x^{\prime}\right|<|x|$. Also, $f^{a_{3}-a_{1}}(x)=f^{b_{2}-a_{1}}(x)$. If $x^{\prime}=\varepsilon$, it follows immediately that $w$ is an alternating $[f, x]$-repetition.

So, assume $x^{\prime} \neq \varepsilon$. Looking at the words occurring around the border between $f^{a_{1}}(w)$ and $f^{a_{2}}(w)$, we get that $f^{a_{1}}\left(x^{\prime}\right) f^{a_{2}}\left(x^{\prime}\right) f^{a_{2}+i_{k}}(x)=f^{b_{1}+i_{k}}(x) f^{b_{1}}\left(x^{\prime}\right) f^{b_{2}-a_{1}}\left(x^{\prime}\right)$.

580 As $f^{a_{3}-a_{1}}(x)=f^{b_{2}-a_{1}}(x)$ (so the image of $x$ under $f$ iterated an even number of times equals the image of $x$ under $f$ iterated an odd number of times) we get that there exists an even number $e_{1}$ such that $f^{e_{1}}(x)=f^{a_{2}+i_{k}}(x)$; for $e_{2}=b_{1}+i_{k}$, we get $f^{a_{1}}\left(x^{\prime}\right) f^{a_{2}}\left(x^{\prime}\right) f^{e_{1}}(x)=f^{e_{2}}(x) f^{b_{1}}\left(x^{\prime}\right) f^{b_{2}-a_{1}}\left(x^{\prime}\right)$. Using Lemma 3. as $f(x)$ is a prefix of $f^{a_{1}}\left(x^{\prime}\right) f^{a_{2}}\left(x^{\prime}\right) f^{e_{1}}(x)$, we get that $f^{e_{1}}(x)=f^{j_{1}}\left(x^{\prime}\right) f^{j_{2}}\left(x^{\prime}\right) \cdots f^{J_{2 p-3}}\left(x^{\prime}\right) f^{j_{2 p-2}}\left(x^{\prime}\right) x^{\prime \prime}$, where $x^{\prime \prime}$ is a prefix, of length less than $2\left|x^{\prime}\right|$, of a word $f^{j_{2 p-1}}\left(x^{\prime}\right) f^{j_{2 p}}\left(x^{\prime}\right)$, and $j_{2 t+1} \equiv_{2}$ $0, j_{2 t+2} \equiv_{2} 1$, for $1 \leq t \leq p-1$.

Once again, there are several cases to be considered. If $x^{\prime \prime}=\varepsilon$, then it follows immediately that $x$ and $w=f^{i_{1}}(x) \ldots f^{i_{k}}(x) x^{\prime}$ are alternating $\left[f, x^{\prime}\right]$-repetitions (recall all $i_{1}, \ldots, i_{k}$ are even). If $x^{\prime \prime}=f^{j_{2 p-1}}\left(x^{\prime}\right)$ then

$$
f^{e_{1}}(x)=f^{j_{1}}\left(x^{\prime}\right) f^{j_{2}}\left(x^{\prime}\right) \cdots f^{j_{2 p-3}}\left(x^{\prime}\right) f^{j_{2 p-2}}\left(x^{\prime}\right) f^{j_{2 p-1}}\left(x^{\prime}\right)
$$

with $j_{1}$ and $j_{2 p-1}$ both even. As there exists an odd number $o_{1}$ such that $x=f^{o_{1}}(x)$, it follows that there exists an odd number $o_{2}$ such that $x^{\prime}=f^{o_{2}}\left(x^{\prime}\right)$. Therefore, both $x$ and $w$ can be expressed as $\left[f, x^{\prime}\right]$-repetitions. So, in the following $x^{\prime \prime}$ is a prefix of $f^{j_{2 p-1}}\left(x^{\prime}\right) f^{j_{2 p}}\left(x^{\prime}\right)$ that has neither length 0 nor $\left|x^{\prime}\right|$. We then get that $f^{b_{1}}\left(x^{\prime}\right) f^{b_{2}-a_{1}}\left(x^{\prime}\right)$ occurs as a suffix of $f^{j_{2 p-3}}\left(x^{\prime}\right) f^{j_{2 p-2}}\left(x^{\prime}\right) x^{\prime \prime}$, which, at its turn is a (strict) factor of $f^{j_{2 p-3}}\left(x^{\prime}\right) f^{j_{2 p-2}}\left(x^{\prime}\right) f^{j_{2 p-1}}\left(x^{\prime}\right) f^{j_{2 p}}\left(x^{\prime}\right)$. In both cases, as $b_{1}$ and $b_{2}$ have different parity and $j_{2 p-3}, j_{2 p-1}$ are even and $j_{2 p-2}, j_{2 p}$ are odd, it follows by the induction hypothesis that $x^{\prime}$ and $x^{\prime \prime}$ are alternating $[f, s]$-repetitions for some word $s$. As $x^{\prime \prime}$ is a prefix of ${ }_{595} f^{j_{2 p-1}}\left(x^{\prime}\right) f^{j_{2 p}}\left(x^{\prime}\right)$ then the alternation of the parity of the exponents of $s$ in the decomposition of $x^{\prime \prime}$ must the same as the corresponding alternation in the decomposition of $f^{j_{2 p-1}}\left(x^{\prime}\right) f^{j_{2 p}}\left(x^{\prime}\right)$. Therefore, $x$ is an alternating $[f, s]$-repetition. Because there exists an odd number $o_{1}$ such that $x=f^{o_{1}}(x)$ and $w=f^{i_{1}}(x) \ldots f^{i_{k}}(x) x^{\prime}$ it follows that there exists a decomposition of both $w$ and $x w$ as an alternating $[f, s]$-repetition. 
It is a trivial remark that if any of the equations from the statement of Lemma 15 has only solutions that are alternating $[f]$-repetitions, then the same holds for all the other three equations. Therefore, all equations from class $(c 9)$ have only solutions which are alternating $[f]$-repetitions.

The fact that class $(c 6)$ is also repetition-enforcing follows now.

Lemma 20. Class (c6) is repetition-enforcing.

Proof. Consider the equation $E: f^{a_{1}}(w) f^{a_{2}}(w) f^{a_{3}}(w)=x f^{b_{1}}(w) f^{b_{2}}(w) y$ corresponding to the representative of class $(c 6)$. Then $y$ is a suffix of $f^{a_{3}}(w)$. Thus $f^{b_{2}-a_{3}}(y)$ is a prefix of $f^{b_{2}}(w)$ (as $b_{2}-a_{3}$ is odd and $f^{b_{2}}(w)=f^{b_{2}-a_{3}}\left(f^{a_{3}}(w)\right)$ ). By the alignment of $f^{b_{2}}(w)$ inside $f^{a_{2}}(w) f^{a_{3}}(w)$, It follows that $f^{b_{2}-a_{3}}(y)$ is a suffix of $f^{a_{2}}(w)$. Therefore, $y$ is a prefix of $f^{a_{2}+a_{3}-b_{2}}(w)$ and $a_{2}+a_{3}-b_{2}$ is odd. Therefore, we get that $f^{a_{2}}(w) f^{a_{3}}(w)$ occurs inside $f^{b_{1}}(w) f^{b_{2}}(w) f^{a_{2}+a_{3}-b_{2}}(w)$, which leads to an equation represented by $(o, e, o \mid e, o)$, so from class $(c 9)$. Such equations are repetition enforcing, by Lemma 20

To conclude this section we propose a series of applications of our repetition enforcing results. In the first one, a repetition enforcing result in the style of Fine and Wilf's theorem is presented.

Theorem 6. Let $u, v \in \Sigma^{+}$such that $|u|<|v|$. Let $f$ be an antimorphic permutation of $\Sigma$ and $\alpha=f^{i_{1}}(u) f^{i_{2}}(u) \cdots f^{i_{k}}(u), \beta=f^{j_{1}}(v) f^{j_{2}}(v) \cdots f^{j_{p}}(v)$ be two words such that: $k, p \geq 3, j_{t} \not_{2} j_{t+1}$ for all $1 \leq t \leq p-1$, and the common prefix of $\alpha$ and $\beta$ is longer than $2|v|+|u|$. Then there exists $\gamma \in \Sigma^{+}$such that $v, u \in\left\{f^{i}(\gamma) \mid 0 \leq i \leq \operatorname{2ord}(f)\right\}^{+}$.

Proof. First of all, it is equivalent to show that if $u$ is $[f]$-primitive, then $v \in\left\{f^{i}(u) \mid\right.$ $0 \leq i \leq 2 \operatorname{ord}(f)\}^{+}$.

If $|u|$ divides $|v|$, the result follows immediately. If $|u|$ divides $2|v|$ but $|u|$ does not divide $|v|$ then, looking at the $f^{i}(u)$ overlapping the border between $f^{j_{1}}(v)$ and $f^{j_{2}}(v)$ and considering that $j_{1}$ and $j_{2}$ have distinct parity, we get that $u=\gamma f^{o_{1}}(\gamma)$ for some word $\gamma$ and odd number $o_{1}$. This is a contradiction.

If $|u|$ does not divide $2|v|$, it follows that $f^{j_{2}}(v)$ ends with $f^{j_{2}-j_{1}}(u)$ and $f^{j_{3}}(v)$ starts with $f^{j_{3}-j_{1}}(u)$ (because $j_{2}-j_{1}$ has a different parity than $j_{2}$, while $j_{2}-j_{1}$ has the same parity as $\left.j_{1}\right)$. So $\beta$ contains $f^{j_{2}-j_{1}}(u) f^{j_{3}-j_{1}}(u)$; moreover, as $|u|$ does not divide $2|v|$, and $\alpha$ and $\beta$ have a common prefix of length at least $2|v|+|u|$, this factor $f^{j_{2}-j_{1}}(u) f^{j_{3}-j_{1}}(u)$ occurs strictly inside a factor $f^{i}(u) f^{i^{\prime}}(u) f^{i^{\prime \prime}}(u)$ of $\alpha$. As $j_{2}-j_{1}$ and $j_{3}-j_{1}$ have different parity, this is an equation from one of the classes (c2), (c5), (c6) or (c9). In all cases, it follows that there exists $\gamma \in \Sigma^{+},|\gamma|<|u|$, such that $u \in\left\{f^{i}(\gamma) \mid 0 \leq i \leq 2 \operatorname{ord}(f)\right\}^{+}$, again a contradiction.

635 This concludes the proof.

The second application shows that an extension of the Lyndon-Schützenberger equation is repetition enforcing.

Theorem 7. Let $f$ be an antimorphic permutation of an alphabet $\Sigma$. Consider the equality:

$$
f^{i_{1}}(u) \ldots f^{i_{r}}(u) f^{j_{1}}(v) \ldots f^{j_{s}}(v)=f^{k_{1}}(w) \ldots f^{k_{t}}(w),
$$


with $r, s \geq 3, t \geq 6$, and $i_{p} \not_{2} i_{p+1}$ for $1 \leq p \leq r-1, j_{p} \not_{2} j_{p+1}$ for $1 \leq p \leq s-1$, $k_{p} \equiv_{2} k_{p+1}$ for $1 \leq p \leq t-1$. Then there exists $\gamma$ such that $u, v, w \in\left\{f^{i}(\gamma) \mid 0 \leq i \leq\right.$ $\operatorname{2ord}(f)\}^{+}$.

PROOF. This proof is split into two parts. Firstly we reduce the given equation to another one of the form $f^{i_{1}}(u) \ldots f^{i_{r}}(u)=f^{k_{1}^{\prime}}(x) \ldots f^{k_{\ell}^{\prime}}(x)$. Afterwards we investigate this equation.

Because $t$ is greater than or equal to 6 it means that at least one of the factors repetitions. In all cases above, the original equation is reduced, once again, to showing that if $f^{i_{1}}(u) \ldots f^{i_{r}}(u)=f^{k_{1}^{\prime}}(x) \ldots f^{k_{\ell}^{\prime}}(x)$, where $k_{p}^{\prime} \not_{2} k_{p+1}^{\prime}$ for $1 \leq p \leq \ell-1$ and $w, v \in\left\{f^{i}(x) \mid 0 \leq i \leq 2 \operatorname{ord}(f)\right\}^{+}$, then there exists $\gamma$ such that $u, x \in\left\{f^{i}(\gamma) \mid 0 \leq\right.$ $i \leq \operatorname{2ord}(f)\}^{+}$.

675 So, it remains to deal with this problem: assume $f^{i_{1}}(u) \ldots f^{i_{r}}(u)=f^{k_{1}^{\prime}}(x) \ldots f^{k^{\prime}}(x)$, where $k_{p}^{\prime} \not \equiv_{2} k_{p+1}^{\prime}$ for $1 \leq p \leq \ell-1$ and $w, v \in\left\{f^{i}(x) \mid 0 \leq i \leq 2 \operatorname{ord}(f)\right\}^{+}$; show that there exists $\gamma$ such that $u, x \in\left\{f^{i}(\gamma) \mid 0 \leq i \leq 2 \operatorname{ord}(f)\right\}^{+}$. But this problem follows just like the cases discussed above. If $\ell=1$ or $\ell=2$, then the conclusion is immediate. If $\ell \geq 3$, then we can apply, e.g., Theorem 7 to reach the conclusion. 


\section{Further Directions}

In this paper we presented a series of equations on words whose solutions are necessarily repetitions under anti-/morphic permutations. The main problem that still remains open is to characterise exactly the triples $(r, s, t)$ for which the equation

$$
f^{i_{1}}(u) \ldots f^{i_{r}}(u) f^{j_{1}}(v) \ldots f^{j_{s}}(v)=f^{k_{1}}(w) \ldots f^{k_{t}}(w),
$$

with $f$ antimorphic permutation, is repetition-enforcing. While Theorem 5 shows that the classical result of Lyndon and Schützenberger is preserved in the generalised case of morphic permutations, we expect that in the case of antimorphic permutations the results obtained in [9] for restricted case of antimorphic involutions should still hold.

\section{Acknowledgements}

A preliminary version of this paper was presented at the 11th International Conference on Combinatorics on Words, WORDS 2017. We gratefully acknowledge the anonymous referees whose comments and suggestions helped us in improving the presentation of this paper. Florin Manea's work was supported by the Deutsche Forschungsgemeinschaft (DFG) grant 389613931.

\section{References}

[1] D. Gusfield, Algorithms on Strings, Trees, and Sequences - Computer Science and Computational Biology, Cambridge University Press, 1997.

[2] M. Lothaire, Applied combinatorics on words, Cambridge University Press, 2005.

[3] M. Lothaire, Combinatorics on words, Cambridge University Press, 1997.

[4] N. J. Fine, H. S. Wilf, Uniqueness theorems for periodic functions, Proc. Amer. Math. Soc. 16 (1965) 109-114.

[5] R. C. Lyndon, M.-P. Schützenberger, The equation $a^{m}=b^{n} c^{p}$ in a free group, Michigan Math. J. 9 (4) (1962) 289-298.

[6] E. Czeizler, L. Kari, S. Seki, On a special class of primitive words, in: Proc. MFCS 2008, Vol. 5162 of LNCS, Springer, 2008, pp. 265-277.

[7] E. Czeizler, L. Kari, S. Seki, On a special class of primitive words, Theoret. Comput. Sci. 411 (3) (2010) 617-630.

[8] E. Czeizler, E. Czeizler, L. Kari, S. Seki, An extension of the LyndonSchützenberger result to pseudoperiodic words, Inform. and Comput. 209 (2011) 717-730.

[9] F. Manea, M. Müller, D. Nowotka, S. Seki, The extended equation of Lyndon and Schützenberger, J. Comput. Syst. Sci. 85 (2017) 132-167. 
[10] F. Manea, R. Mercaş, D. Nowotka, Fine and Wilf's Theorem and pseudorepetitions, in: Proc. MFCS 2012, Vol. 7464 of LNCS, Springer, 2012, pp. 668680.

[11] F. Manea, M. Müller, D. Nowotka, Cubic patterns with permutations, J. Comput. Syst. Sci. 81 (7) (2015) 1298-1310.

[12] J. D. Currie, F. Manea, D. Nowotka, Unary patterns with permutations, in: Proc. DLT 2015, Vol. 9168 of LNCS, Springer, 2015, pp. 191-202.

[13] P. Gawrychowski, F. Manea, R. Mercaş, D. Nowotka, C. Tiseanu, Finding pseudorepetitions, in: Proc. STACS 2013, Vol. 20 of LIPIcs, 2013, pp. 257-268.

[14] P. Gawrychowski, F. Manea, D. Nowotka, Discovering hidden repetitions in words, in: Proc. CiE 2013, Vol. 7921 of LNCS, 2013, pp. 210-219.

[15] P. Gawrychowski, F. Manea, R. Mercas, D. Nowotka, Hide and seek with repetitions J. Comput. Syst. Sci. 101 (2019) 42-67. doi:10.1016/j.jcss. 2018. 10.004

URL https://doi.org/10.1016/j.jcss.2018.10.004

[16] E. Chiniforooshan, L. Kari, Z. Xu, Pseudopower avoidance, Fund. Inform. 114 (1) (2012) 55-72.

[17] P. Gawrychowski, F. Manea, D. Nowotka, Testing generalised freeness of words, in: Proc. STACS 2014, Vol. 25 of LIPIcs, 2014, pp. 337-349.

[18] Z. Xu, A minimal periods algorithm with applications, in: Proc. CPM 2010, Vol. 6129 of LNCS, Springer, 2010, pp. 51-62.

730 [19] L. Kari, B. Masson, S. Seki, Properties of pseudo-primitive words and their applications, Internat. J. Found. Comput. Sci. 22 (2) (2011) 447-471. 